TRANSACTIONS OF THE

AMERICAN MATHEMATICAL SOCIETY

Volume 355, Number 1, Pages 99-117

S 0002-9947(02)03051-9

Article electronically published on September 5, 2002

\title{
ON THE JACOBI GROUP AND THE MAPPING CLASS GROUP OF $S^{3} \times S^{3}$
}

\author{
NIKOLAI A. KRYLOV
}

\begin{abstract}
The paper contains a proof that the mapping class group of the manifold $S^{3} \times S^{3}$ is isomorphic to a central extension of the (full) Jacobi group $\Gamma^{J}$ by the group of 7-dimensional homotopy spheres. Using a presentation of the group $\Gamma^{J}$ and the $\mu$-invariant of the homotopy spheres, we give a presentation of this mapping class group with generators and defining relations. We also compute the cohomology of the group $\Gamma^{J}$ and determine 2-cocycles that correspond to the mapping class group of $S^{3} \times S^{3}$.
\end{abstract}

\section{INTRODUCTION}

The central theme of this paper is the group of isotopy classes of orientationpreserving diffeomorphisms on $S^{3} \times S^{3}$. We denote this group by $\pi_{0} \operatorname{Diff}\left(S^{3} \times S^{3}\right)$. In general, the group of isotopy classes of orientation-preserving diffeomorphisms on a closed oriented smooth manifold $M$ will be denoted by $\pi_{0} D i f f\left(S^{3} \times S^{3}\right)$ and called the mapping class group of $M$ by analogy with the 2-dimensional case.

The article consists of two parts. Our goal in the first part will be to give a presentation of the mapping class group of $S^{3} \times S^{3}$ with generators and defining relations. The main step in this direction is Theorem 1 , where we prove that $\pi_{0} \operatorname{Diff}\left(S^{3} \times S^{3}\right)$ is a central extension of the (full) Jacobi group $\Gamma^{J}$ by the group of 7-dimensional homotopy spheres $\Theta_{7}$. The second part is concerned with the cohomology group $H^{2}\left(\Gamma^{J}, \mathbb{Z}_{28}\right)$. We show that this group is isomorphic to $\mathbb{Z}_{28} \oplus$ $\mathbb{Z}_{4} \oplus \mathbb{Z}_{2}$ and determine a 2-cocycle that corresponds to $\pi_{0} \operatorname{Diff}\left(S^{3} \times S^{3}\right)$.

The mapping class group of $S^{3} \times S^{3}$ was also studied by D. Fried in [10. In particular, he proves that the group of the isotopy classes of diffeomorphisms that act trivially on $H_{3}\left(S^{3} \times S^{3}\right)$ is isomorphic to $H_{\mathbb{Z}} / 28 \mathbb{Z}$, where $H_{\mathbb{Z}}$ is the group of the integral upper unitriangular $3 \times 3$ matrices and $\mathbb{Z}$ is the center of $H_{\mathbb{Z}}$. The proof uses the $\mu$-invariant of J. Eells and N. Kuiper 7 to show that $v \otimes v$ goes to the generator of $\Theta_{7}$ under the bilinear pairing $\pi_{3}(S O(3)) \otimes \pi_{3}(S O(3)) \longrightarrow \Theta_{7}$ defined by J. Milnor [17] ( $v$ here is the generator of $\pi_{3}(S O(3)$ ), given by the conjugation action of unit quaternions). We give details in section 1.3. Moreover, Fried (see Theorem 2) derives the exact sequence:

$$
1 \longrightarrow S \longrightarrow \Phi_{2} \longrightarrow \pi_{0} \operatorname{Diff}\left(S^{3} \times S^{3}\right) \longrightarrow 1
$$

where $\Phi_{2}$ is the automorphism group of $F_{2}$, the free group on two generators, and the kernel $S \subset F_{2}^{\prime}=\left[F_{2}, F_{2}\right] \subset F_{2} \subset \Phi_{2}$ is characterized by the commutative

Received by the editors July 18, 2001 and, in revised form, March 15, 2002.

2000 Mathematics Subject Classification. Primary 57R50, 57R52; Secondary $20 J 06$. 
diagram:

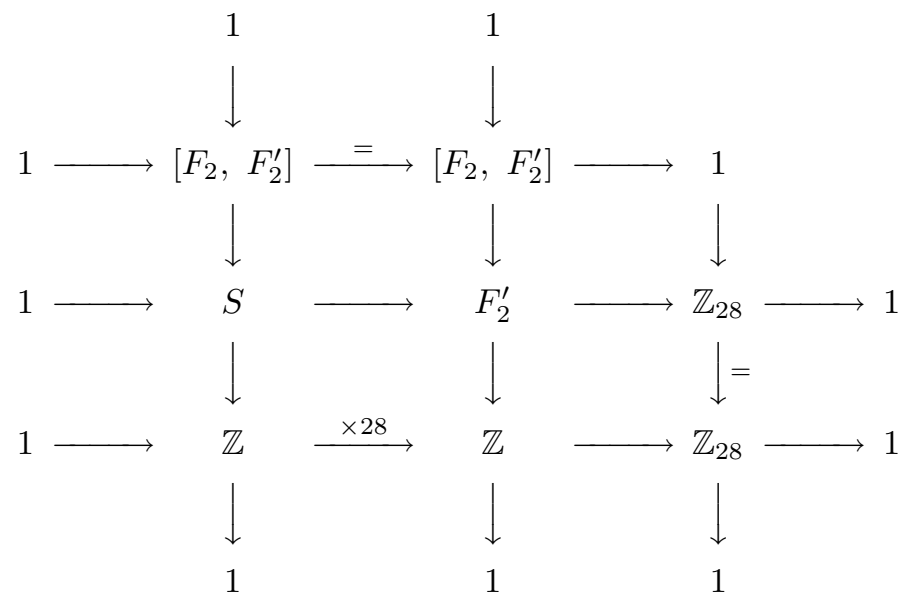

In the last section, we show that a generator of $H^{2}\left(S L_{2}(\mathbb{Z}), \mathbb{Z}_{28}\right) \cong \mathbb{Z}_{4}$ corresponds to the mapping class group of $S^{3} \times S^{3}$. This implies, in particular, that the natural map $\pi_{0} \operatorname{Diff}\left(S^{3} \times S^{3}\right) \longrightarrow S L_{2}(\mathbb{Z})$ does not split (cf. [10], $\left.\S 2\right)$.

\section{A presentation of the group $\pi_{0} \operatorname{Diff}\left(S^{3} \times S^{3}\right)$}

For a closed smooth oriented $(k-1)$-connected almost-parallelizable manifold $M^{2 k}, k \geq 2$, the group $\pi_{0} \operatorname{Diff}(M)$ has been computed in terms of exact sequences by M. Kreck [14] (see also [18], [19]). We will begin by recalling his results in the first section. Next we will show that there is an isomorphism between the factor group $\pi_{0} \operatorname{Diff}\left(S^{3} \times S^{3}\right) / \Theta_{7}$ and the (full) Jacobi group $\Gamma^{J}$. In the last section of this part, we give a presentation of $\pi_{0} \operatorname{Diff}\left(S^{3} \times S^{3}\right)$ with generators and defining relations (Theorem 3). All diffeomorphisms are assumed to be orientationpreserving and integer coefficients are understood for all homology and cohomology groups.

1.1. Exact sequences of Kreck. Our focus in this paragraph is to review results of Kreck [14]. First we recall some of the definitions and notation. From now on by a manifold $M$ we will mean a closed oriented differentiable $(k-1)$-connected almost-parallelizable $2 k$-manifold.

Denote by Aut $H_{k}(M)$ the group of automorphisms of $H_{k}(M, \mathbb{Z})$ preserving the intersection form on $M$ and (for $k \geq 3$ ) commuting with the function $\alpha: H_{k}(M)$ $\longrightarrow \pi_{k-1}(S O(k))$, which is defined as follows. Represent $x \in H_{k}(M)$ by an embedded sphere $S^{k} \hookrightarrow M$. Then function $\alpha$ assigns to $x$ the classifying map of the corresponding normal bundle. Any diffeomorphism $f \in \operatorname{Diff}(M)$ induces map $f_{*}$ that lies in Aut $H_{k}(M)$. This gives a homomorphism

$$
\kappa: \pi_{0} \operatorname{Diff}(M) \longrightarrow \text { Aut } H_{k}(M), \quad[f] \longmapsto f_{*}
$$

The kernel of $\kappa$ is denoted by $\pi_{0} S$ Diff $(M)$. For elements of $\pi_{0} S$ Diff $(M)$, Kreck defines the following invariant. Choose again a sphere $S^{k} \hookrightarrow M$ that represents an element $x \in H_{k}(M)$. Since $[f] \in \pi_{0} S \operatorname{Diff}(M)$, we can assume (for $k>3$ ) that $\left.f\right|_{S^{k}}=I d$. The stable normal bundle $\nu\left(S^{k}\right) \oplus 1$ of $S^{k}$ in $M$ is trivial and we can choose some trivialization $\tau: \nu\left(S^{k}\right) \oplus 1 \longrightarrow S^{k} \times \mathbb{R}^{k+1}$. Clearly, the differential of $f$ leaves the tangent bundle of $S^{k}$ invariant and hence induces an automorphism 
of the normal bundle $\nu\left(S^{k}\right) \oplus 1$. At each point $t \in S^{k}$, this automorphism gives (via trivialization $\tau$ ) an element $\mathcal{P}_{t}$ of the group $S O(k+1)$ and hence we get an element $\mathcal{P} \in \pi_{k}(S O(k+1))$. It is obvious that $\mathcal{P}$ lies in the image of the map $S: \pi_{k}(S O(k)) \longrightarrow \pi_{k}(S O(k+1))$ induced by the inclusion $S O(k) \hookrightarrow S O(k+$ 1). It is a standard fact that element $\mathcal{P}$ does not depend on the trivialization $\tau$ of the normal bundle $\nu\left(S^{k}\right) \oplus 1$. This construction leads to a well-defined homomorphism (cf. Lemmas 1,2 of [14])

$$
\chi: \pi_{0} S \operatorname{Diff}(M) \longrightarrow \operatorname{Hom}\left(H_{k}(M), S \pi_{k}(S O(k))\right)
$$

For $k \equiv 3(\bmod 4)$, the group $S \pi_{k}(S O(k))$ is isomorphic to the cyclic group $\mathbb{Z}$ and hence we can identify $\operatorname{Hom}\left(H_{k}(M), S \pi_{k}(S O(k))\right)$ with the cohomology group $H^{k}(M)$. In this case, one can describe $\chi(f)$ by the Pontrjagin class of the mapping torus $M_{f}$, but we will not use this description here and so we omit the details. The following theorem is due to Kreck (see [14]).

Theorem. If $M^{2 k}(k \geq 3)$ bounds a framed manifold, then the following sequences are exact:

$$
\begin{gathered}
0 \longrightarrow \pi_{0} S \operatorname{Diff}(M) \longrightarrow \pi_{0} \operatorname{Diff}(M) \stackrel{\kappa}{\longrightarrow} \text { Aut } H_{k}(M) \longrightarrow 0, \\
0 \longrightarrow \Theta_{2 k+1} \stackrel{\iota}{\longrightarrow} \pi_{0} S \operatorname{Diff}(M) \stackrel{\chi}{\longrightarrow} \operatorname{Hom}\left(H_{k}(M), S \pi_{k}(S O(k))\right) \longrightarrow 0 .
\end{gathered}
$$

Remark. If we consider an $M^{2 k}$ that does not bound a framed manifold, then $\operatorname{Ker}(\chi)$ will be a factor group $\Theta_{2 k+1} / \Sigma_{M}$ instead of the whole group of homotopy spheres. Since $S^{3} \times S^{3}$ is the boundary of $S^{3} \times D^{4}$, we have $\Sigma_{M}=0$ (cf. Lemma 3 of [14).

Map $\iota$ is defined as follows. Present a homotopy sphere $\Sigma \in \Theta_{2 k+1}$ as a union $D^{2 k+1} \bigcup_{f} D^{2 k+1}$ and assume that $f=i d$ on a neighbourhood of the lower hemisphere $D_{-}^{2 k} \subset S^{2 k}$. Then $\iota(\Sigma)$ is the class of diffeomorphisms on $M$ that is identity outside an embedded disk in $M$ and is equal to $\left.f\right|_{D_{+}^{2 k}}$ on this disk.

It follows from the second exact sequence that $\pi_{0} S \operatorname{Diff}\left(S^{3} \times S^{3}\right) / \Theta_{7}$ is isomorphic to $\mathbb{Z} \oplus \mathbb{Z}$. Note also that Aut $H_{3}\left(S^{3} \times S^{3}\right) \cong S L_{2}(\mathbb{Z})$ (cf. Theorem I of [18]). Exact sequences (11) and (2) induce the following short exact sequence:

$$
0 \longrightarrow \mathbb{Z} \oplus \mathbb{Z} \longrightarrow \pi_{0} \operatorname{Diff}\left(S^{3} \times S^{3}\right) / \Theta_{7} \longrightarrow S L_{2}(\mathbb{Z}) \longrightarrow 0 .
$$

In the next section, we prove that this exact sequence splits and the factor group $\pi_{0} \operatorname{Diff}\left(S^{3} \times S^{3}\right) / \Theta_{7}$ is isomorphic to the (full) Jacobi group $\Gamma^{J}$ (cf. [10], §2).

1.2. Splitting of the exact sequence. The (full) Jacobi group $\Gamma^{J}$ is a semidirect product of the modular group with the direct sum $\mathbb{Z} \oplus \mathbb{Z}$. More precisely (cf. [8], $\S$ I.1), $\Gamma^{J} \stackrel{\text { def }}{=} S L_{2}(\mathbb{Z}) \ltimes \mathbb{Z}^{2}=$ the set of pairs $(M, X)$ with $M \in S L_{2}(\mathbb{Z}), X \in \mathbb{Z} \oplus \mathbb{Z}$ and the group law $(M, X) \cdot\left(M^{\prime}, X^{\prime}\right)=\left(M M^{\prime}, X M^{\prime}+X^{\prime}\right)$ (notice that vectors are written as row vectors, i.e., $S L_{2}(\mathbb{Z})$ acts on the right). It is interesting to note that $\Gamma^{J}$ first came up in the theory of Jacobi forms (see [8]). We will need

Lemma 1. $\Gamma^{J}$ admits the following presentation: $\langle y, u, a, b|$ yuy $=u y u,(y u y)^{4}=$ $\left.i d, a b=b a, a y=y a b, a u=u a, b y=y b, b u=u b a^{-1}\right\rangle$.

Proof. $S L_{2}(\mathbb{Z})$ has a presentation: $\left\langle y, u \mid y u y=u y u,(y u y)^{4}=i d\right\rangle$ (see, for example, [2]) where $y$ and $u$ correspond to matrices $\left(\begin{array}{ll}1 & 1 \\ 0 & 1\end{array}\right),\left(\begin{array}{cc}1 & 0 \\ -1 & 1\end{array}\right)$ respectively. (It is a classical fact that $S L_{2}(\mathbb{Z}) \cong \mathbb{Z}_{4} *_{\mathbb{Z}_{2}} \mathbb{Z}_{6}$. Hence $S L_{2}(\mathbb{Z})$ has a presentation: 
$\left\langle x, z \mid x^{4}=i d, x^{2}=z^{3}\right\rangle$. One can use a map $f: f(z)=y u, f(x)=(y u y)^{-1}$ to show that these two presentations define isomorphic groups.) By definition of $\Gamma^{J}$, the following sequence is exact:

$$
0 \longrightarrow \mathbb{Z}^{2} \longrightarrow \Gamma^{J} \longrightarrow S L_{2}(\mathbb{Z}) \longrightarrow 0 .
$$

Consider a homomorphism $\alpha: S L_{2}(\mathbb{Z}) \longrightarrow \Gamma^{J}$ defined by the formulas: $\alpha(y) \stackrel{\text { def }}{=}$ $(y,(0,0)), \alpha(u) \stackrel{\text { def }}{=}(u,(0,0))$. If we denote elements $(y,(0,0)) ;(u,(0,0)) ;(i d,(1,0))$; $(i d,(0,1))$ by $y, u, a$ and $b$ respectively, we see that these elements $y, u, a, b$ generate $\Gamma^{J}$ and the relations yuy $=u y u,(y u y)^{4}=i d, a b=b a$ are satisfied. To find all defining relations for $\Gamma^{J}$, we need to find how $S L_{2}(\mathbb{Z})$ acts on the generators $a$ and $b$ of $\mathbb{Z}^{2}$ by conjugation. First note that $a b=(i d,(1,0)) \cdot(i d,(0,1))=(i d,(1,1))$ and $b a^{-1}=(i d,(0,1)) \cdot(i d,(-1,0))=(i d,(-1,1))$. Hence,

$$
\begin{aligned}
a y & =(i d,(1,0)) \cdot(y,(0,0))=(y,(1,0) \cdot y+(0,0))=(y,(1,1)), \\
y a b & =(y,(0,0)) \cdot(i d,(1,1))=(y,(1,1)) \Rightarrow a y=y a b, \\
a u & =(i d,(1,0)) \cdot(u,(0,0))=(u,(1,0) \cdot u+(0,0))=(u,(1,0)), \\
u a & =(u,(0,0)) \cdot(i d,(1,0))=(u,(1,0)) \Rightarrow a u=u a, \\
b y & =(i d,(0,1)) \cdot(y,(0,0))=(y,(0,1) \cdot y+(0,0))=(y,(0,1)), \\
y b & =(y,(0,0)) \cdot(i d,(0,1))=(y,(0,1)) \Rightarrow b y=y b, \\
b u & =(i d,(0,1)) \cdot(u,(0,0))=(u,(0,1) \cdot u+(0,0))=(u,(-1,1)), \\
u b a^{-1} & =(u,(0,0)) \cdot(i d,(-1,1))=(u,(-1,1)) \Rightarrow b u=u b a^{-1} .
\end{aligned}
$$

Remark. A different presentation of this group can be found in [6] (cf. also Lemma 6 below).

Consider now the standard sphere $S^{3}$ in Euclidean four-space $\mathbb{R}^{4}$, given by the equation: $x_{0}^{2}+x_{1}^{2}+x_{2}^{2}+x_{3}^{2}=1$. This sphere can be identified with the special unitary group $S U(2)$, which is also known as the group of unit quaternions. The group structure on $S^{3}$ induces the group structure on the product $S^{3} \times S^{3}$. If we denote elements of the group $S^{3}$ by $s$ and $t$, we will write $(s, t)$ to denote the corresponding element of the group $S^{3} \times S^{3}$. The product of two elements $(s, t)$ and $\left(s^{\prime}, t^{\prime}\right)$ will be the pair $\left(s s^{\prime}, t t^{\prime}\right)$ with quaternion multiplication understood.

Theorem 1. The factor group $\pi_{0} D i f f\left(S^{3} \times S^{3}\right) / \Theta_{7}$ is isomorphic to the Jacobi group $\Gamma^{J}$.

Proof. We will give a presentation of the factor group $\pi_{0} \operatorname{Diff}\left(S^{3} \times S^{3}\right) / \Theta_{7}$ that coincides with the above presentation of $\Gamma^{J}$. By $y$ and $u$ we denote the generators of $S L_{2}(\mathbb{Z})$ as above. Consider isotopy classes $[Y]$ and $[U]$ of the following diffeomorphisms of $S^{3} \times S^{3}$ :

$$
Y:(s, t) \longmapsto(s, s t), U:(s, t) \longmapsto\left(t^{-1} s, t\right) .
$$

Define map $\beta: S L_{2}(\mathbb{Z}) \rightarrow \pi_{0} \operatorname{Diff}\left(S^{3} \times S^{3}\right)$ by the identities: $\beta(y) \stackrel{\text { def }}{=}[Y], \beta(u) \stackrel{\text { def }}{=}$ $[U]$ and extend it linearly to the whole group $S L_{2}(\mathbb{Z})$. We will show that $\beta$ is a well-defined homomorphism from $S L_{2}(\mathbb{Z})$ to $\pi_{0} \operatorname{Diff}\left(S^{3} \times S^{3}\right) / \Theta_{7}$. First we check 
that $Y U Y=U Y U$ :

$$
\begin{aligned}
& Y U Y:(s, t) \stackrel{Y}{\longmapsto}(s, s t) \stackrel{U}{\longmapsto}\left((s t)^{-1} s, s t\right)=\left(t^{-1}, s t\right) \stackrel{Y}{\longmapsto}\left(t^{-1}, t^{-1} s t\right), \\
& U Y U:(s, t) \stackrel{U}{\longmapsto}\left(t^{-1} s, t\right) \stackrel{Y}{\longmapsto}\left(t^{-1} s, t^{-1} s t\right) \stackrel{U}{\longmapsto}\left(t^{-1}, t^{-1} s t\right) .
\end{aligned}
$$

Thus $Y U Y=U Y U$ and hence $[Y][U][Y]=[U][Y][U]$. From now on, we will denote a diffeomorphism and the isotopy class of it by the same capital letter, omitting the brackets. To prove the equality $(Y U Y)^{4}=I d$ ( $I d$ stands for the identity diffeomorphism of $S^{3} \times S^{3}$ ), we will need some auxiliary results. Consider the following diffeomorphisms $A, B \in \operatorname{Diff}\left(S^{3} \times S^{3}\right)$ :

$$
A:(s, t) \longmapsto\left(t s t^{-1}, t\right), \quad B:(s, t) \longmapsto\left(s, s t s^{-1}\right) .
$$

If we choose spheres $S^{3} \times 1$ and $1 \times S^{3}$ as generators of the group $H_{3}\left(S^{3} \times S^{3}\right)$, it is obvious that diffeomorphisms $A$ and $B$ preserve these spheres and act trivially on the homology of $S^{3} \times S^{3}$.

Lemma 2. $\chi(A)$ and $\chi(B)$ generate the group $\left.\operatorname{Hom}\left(H_{3}\left(S^{3} \times S^{3}\right), \mathbb{Z}\right)\right)$.

Proof of lemma. Let us compute $\chi(B)$. Since $S^{3} \times S^{3}$ is a parallelizable manifold and the normal bundle of $S_{1}^{3} \stackrel{\text { def }}{=} S^{3} \times 1$ in $S^{3} \times S^{3}$ is trivial, we need to find an element of the group $\pi_{3}(S O(3))$ that corresponds to the differential of $B$. Take a point $(s, 1) \in S_{1}^{3}$. We can identify the fiber of the normal bundle $\nu\left(S_{1}^{3}\right)$ at $(s, 1)$ with the fiber of the tangent bundle $\tau\left(s \times S^{3}\right)$ at this point. Furthermore, via the projection $\rho_{2}: S^{3} \times S^{3} \longrightarrow 1 \times S^{3}$, we can identify this tangent fiber at $(s, 1)$ with the tangent fiber at $(1,1)$ (Lie algebra $\mathfrak{g}$ of the group $\left.1 \times S^{3}\right)$. Since $\left.B_{s}(t) \stackrel{\text { def }}{=} B\right|_{s \times S^{3}}(s, t)=s t s^{-1}$, the map $s \mapsto d_{(s, 1)} B_{s}$ will correspond to the $a d-$ joint representation $A d: S_{1}^{3} \longrightarrow A u t(\mathfrak{g})$, and $\chi(B)(s, 1)=A d_{s} \in S O(3)$. Thus, $\chi(B)\left(S^{3} \times 1\right)=A d: S^{3} \times 1 \longrightarrow S O(3)$. If we choose an element $T \in \mathfrak{g}$, then it is well known that $A d_{s}(T)=s T s^{-1}$. This map is a generator of the group $\pi_{3}(S O(3))$ (see [15], ch. I, §2) and therefore the isotopy class of the diffeomorphism $B$ is a generator of the group $H^{3}\left(S^{3} \times S^{3}\right)$. In a similar way, one can show that the isotopy class of the diffeomorphism $A$ is the other generator (corresponding to the map $\left.\chi(A)\left(1 \times S^{3}\right): 1 \times S^{3} \longrightarrow S O(3)\right)$ of the group $H^{3}\left(S^{3} \times S^{3}\right)$.

Now we show that

$$
A B=B A, A Y=Y A B, A U=U A, B Y=Y B, B U=U B A^{-1}
$$

in the factor group $\pi_{0} \operatorname{Diff}\left(S^{3} \times S^{3}\right) / \Theta_{7}$. The first equality follows from the results of Kreck since, as we just saw, $A$ and $B$ generate the abelian subgroup of the group $\pi_{0} \operatorname{Diff}\left(S^{3} \times S^{3}\right) / \Theta_{7}$. To prove the other equalities, we use the group structure on 


$$
\begin{aligned}
& S^{3} \times S^{3}: \\
& A Y:(s, t) \stackrel{Y}{\longmapsto}(s, s t) \stackrel{A}{\longmapsto}\left(s t s t^{-1} s^{-1}, s t\right), \\
& Y A B:(s, t) \stackrel{B}{\longmapsto}\left(s, s t s^{-1}\right) \stackrel{A}{\longmapsto}\left(s t s^{-1} s s t^{-1} s^{-1}, s t s^{-1}\right)=\left(s t s t^{-1} s^{-1}, s t s^{-1}\right) \\
& \stackrel{Y}{\longmapsto}\left(s t s t^{-1} s^{-1}, s t s t^{-1} s^{-1} s t s^{-1}\right)=\left(s t s t^{-1} s^{-1}, s t\right) \Rightarrow A Y=Y A B, \\
& A U:(s, t) \stackrel{U}{\longmapsto}\left(t^{-1} s, t\right) \stackrel{A}{\longmapsto}\left(t t^{-1} s t^{-1}, t\right)=\left(s t^{-1}, t\right), \\
& U A:(s, t) \stackrel{A}{\longmapsto}\left(t s t^{-1}, t\right) \stackrel{U}{\longmapsto}\left(t^{-1} t s t^{-1}, t\right)=\left(s t^{-1}, t\right) \Rightarrow A U=U A, \\
& B Y:(s, t) \stackrel{Y}{\longmapsto}(s, s t) \stackrel{B}{\longmapsto}\left(s, s s t s^{-1}\right), \\
& Y B:(s, t) \stackrel{B}{\longmapsto}\left(s, s t s^{-1}\right) \stackrel{Y}{\longmapsto}\left(s, s s t s^{-1}\right) \Rightarrow B Y=Y B, \\
& B U:(s, t) \stackrel{U}{\longmapsto}\left(t^{-1} s, t\right) \stackrel{B}{\longmapsto}\left(t^{-1} s, t^{-1} s t s^{-1} t\right), \\
& U B A^{-1}:(s, t) \stackrel{A^{-1}}{\longmapsto}\left(t^{-1} s t, t\right) \stackrel{B}{\longmapsto}\left(t^{-1} s t, t^{-1} s t t t^{-1} s^{-1} t\right)=\left(t^{-1} s t, t^{-1} s t s^{-1} t\right) \\
& \stackrel{U}{\longmapsto}\left(t^{-1} s t^{-1} s^{-1} t t^{-1} s t, t^{-1} s t s^{-1} t\right)=\left(t^{-1} s, t^{-1} s t s^{-1} t\right) \Rightarrow B U=U B A^{-1}
\end{aligned}
$$

as required.

Claim 1. $\left(B^{-1} Y U Y\right)^{4}=I d, Y U Y B^{-1}=A^{-1} Y U Y, Y U Y A^{-1}=B Y U Y$.

Proof of claim: $B^{-1} Y U Y:(s, t) \stackrel{Y U Y}{\longmapsto}\left(t^{-1}, t^{-1} s t\right) \stackrel{B^{-1}}{\longmapsto}\left(t^{-1}, t t^{-1} s t t^{-1}\right)=\left(t^{-1}, s\right)$ and hence $\left(B^{-1} Y U Y\right)^{4}:(s, t) \longmapsto\left(t^{-1}, s\right) \longmapsto\left(s^{-1}, t^{-1}\right) \longmapsto\left(t, s^{-1}\right) \longmapsto(s, t)$, i.e., $\left(B^{-1} Y U Y\right)^{4}=I d$. Identities $A Y=Y A B, B U=U B A^{-1}, A B=B A$ and $A U=U A$ imply $Y B^{-1} A^{-1}=A^{-1} Y, U B^{-1}=B^{-1} A^{-1} U$. Then from the above equalities we get $Y U Y B^{-1}=Y U B^{-1} Y=Y B^{-1} A^{-1} U Y=A^{-1} Y U Y$. Similarly we see that $Y A^{-1}=B A^{-1} Y, U B=B U A$; hence $Y U Y A^{-1}=Y U B A^{-1} Y=$ $Y B U A A^{-1} Y=Y B U Y=B Y U Y$, which proves the claim.

Using these identities, we can show that $(Y U Y)^{4}=I d$. Indeed,

$$
\begin{aligned}
I d & =\left(B^{-1} Y U Y\right)^{4}=B^{-1} Y U Y B^{-1} Y U Y B^{-1} A^{-1}(Y U Y)^{2} \\
& =B^{-1} Y U Y B^{-1} B Y U Y B^{-1}(Y U Y)^{2}=B^{-1} Y U Y Y U Y B^{-1}(Y U Y)^{2} \\
& =B^{-1} Y U Y A^{-1}(Y U Y)^{3}=(Y U Y)^{4} .
\end{aligned}
$$

It implies that the exact sequence (3) splits, the factor group $\pi_{0} D i f f\left(S^{3} \times S^{3}\right) / \Theta_{7}$ has four generators $Y, U, A, B$ and the following set of defining relations: $Y U Y=$ $U Y U,(Y U Y)^{4}=I d, A B=B A, A Y=Y A B, A U=U A, B Y=Y B, B U=$ $U B A^{-1}$. In particular, the groups $\pi_{0} \operatorname{Diff}\left(S^{3} \times S^{3}\right) / \Theta_{7}$ and $\Gamma^{J}$ have the same presentations and are therefore isomorphic.

Note that diffeomorphisms $A$ and $B$ have been considered by W. Browder ([3], Theorem 6) to give an example of diffeomorphisms of $S^{3} \times S^{3}$ that are homotopic to the identity, but are not pseudo-isotopic to the identity.

1.3. Group $\pi_{0} S \operatorname{Diff}\left(S^{3} \times S^{3}\right)$. In this paragraph, we prove that the group $\pi_{0} S \operatorname{Diff}\left(S^{3} \times S^{3}\right)$ is isomorphic to the group $\mathcal{H}_{28}$ where

$$
\mathcal{H}_{m} \stackrel{\text { def }}{=}\left\{\left(\begin{array}{lll}
1 & a & l \\
0 & 1 & b \\
0 & 0 & 1
\end{array}\right) \mid a, b \in \mathbb{Z} \text { and } l \in \mathbb{Z}_{m}\right\} .
$$


The group $\mathcal{H}_{m}$ also can be described as the factor group of the group $\mathcal{H}$ (upper unitriangular $3 \times 3$ matrices with integer coefficients) by the cyclic subgroup generated by the matrix with $a=b=0$ and $l=m$.

The idea of the proof is to compare the presentations of two groups as we just did above. First we give a presentation of $\pi_{0} S D i f f\left(S^{3} \times S^{3}\right)$. We know already that $H^{3}\left(S^{3} \times S^{3}, \mathbb{Z}\right)$ is generated by $\chi(A)$ and $\chi(B)$. We also know from the exact sequence (2) that $\pi_{0} S \operatorname{Diff}\left(S^{3} \times S^{3}\right)$ is a central extension of the group $\mathbb{Z} \oplus \mathbb{Z}$ by $\Theta_{7} \cong \mathbb{Z}_{28}$ (see [13]). If we denote by $\widehat{\Sigma}$ the generator of $\Theta_{7}$ (by the generator we mean a homotopy 7 -sphere that bounds a parallelizable manifold of signature 8), then we can choose $A, B$ and $\Sigma \stackrel{\text { def }}{=} \iota(\widehat{\Sigma})$ as generators of $\pi_{0} S D$ iff $\left(S^{3} \times S^{3}\right)$. The defining relations clearly will be: $A \Sigma=\Sigma A, B \Sigma=\Sigma B, A B A^{-1} B^{-1}=\Sigma^{k}$, $\Sigma^{28}=1$ for some $k \in \mathbb{Z}_{28}$. So the goal is to figure out what this $k$ is.

We first define a map $\varsigma$ : $\pi_{0} S \operatorname{Diff}\left(S^{3} \times S^{3}\right) \longrightarrow \mathbb{Z}_{28}$ as follows. Take a representative $f \in \operatorname{Diff}\left(S^{3} \times S^{3}\right)$ of a class $[f] \in \pi_{0} S \operatorname{Diff}\left(S^{3} \times S^{3}\right)$.

\section{Definition 1.}

$$
\varsigma([f]) \stackrel{\text { def }}{=} D^{4} \times S^{3} \cup_{f} S^{3} \times D^{4},
$$

where $\cup_{f}$ means identification of a point $(x, y) \in \partial\left(S^{3} \times D^{4}\right)$ with the point $f(x, y) \in$ $\partial\left(D^{4} \times S^{3}\right)$.

Denote by $\Sigma_{f}$ the manifold obtained from $\varsigma([f])$ by smoothing the corners. It is clear that $\Sigma_{f}$ depends only on the isotopy class $[f]$ and not on a specific representative of this class. Note that $\Sigma_{f}$ is a homotopy sphere and $\varsigma$ is a well-defined map from $\pi_{0} S$ Diff $\left(S^{3} \times S^{3}\right)$ to the group $\Theta_{7}$.

Remark. Map $\varsigma$ is the analog of the Birman-Craggs homomorphism from the Torelli group of $S_{g}^{2}$ (2-dimensional surface of genus $g$ ) to $\mathbb{Z}_{2}$. See [12] for the details (cf. also [18], §4).

Lemma 3. The composition $\varsigma \circ \iota$ is the identity map of the group $\Theta_{7}$.

Proof. Take a sphere $\widetilde{\Sigma}_{\phi} \in \Theta_{7}$. We will denote by $\phi$ the diffeomorphism of $S^{3} \times S^{3}$ that is the identity outside an embedded disk $D^{6} \subset S^{3} \times S^{3}$ and corresponds to the element $\iota\left(\widetilde{\Sigma}_{\phi}\right)$ of the mapping class group.

To show that $\varsigma \circ \iota=I d$, it is enough to show that $\widetilde{\Sigma}_{\phi}$ is diffeomorphic to $\Sigma_{\phi}=\varsigma \circ \iota\left(\widetilde{\Sigma}_{\phi}\right)$. We construct an h-cobordism between these two manifolds. Take the handlebody $D^{4} \times S^{3}$ and remove from it an interior disk $D^{7}$. The resulting manifold is denoted by $\widetilde{D^{4} \times S^{3}}$. The boundary components of $\widetilde{D^{4} \times S^{3}}$ are $S^{6}$ and $S^{3} \times S^{3}$. Take disks $D_{+}^{6}$ in these two components and connect them by a tube $D_{+}^{6} \times I$ embedded into $\widetilde{D^{4} \times S^{3}}$. Next extend $\phi$ in an obvious way (by the identity outside the tube) to a diffeomorphism $\Phi$ of $\widetilde{D^{4} \times S^{3}}$. Consider now two manifolds: $D^{5} \times S^{3}$ and $D^{8}$. Present the boundary of $D^{5} \times S^{3}$ as the union:

$$
\partial\left(D^{5} \times S^{3}\right)=S^{4} \times S^{3}=D^{4} \times S^{3} \cup_{S^{3} \times S^{3}} \widetilde{D^{4} \times S^{3}} \cup_{S^{6}} D^{7}
$$

and the boundary of $D^{8}$ as the union:

$$
\partial\left(D^{8}\right)=S^{7}=S^{3} \times D^{4} \cup_{S^{3} \times S^{3}} \widetilde{D^{4} \times S^{3}} \cup_{S^{6}} D^{7} .
$$

Using the diffeomorphism $\Phi$, we can glue $D^{5} \times S^{3}$ and $D^{8}$ together along the common submanifold $\widetilde{D^{4} \times S^{3}}$ to obtain a cobordism (after smoothing the corners) 
$W^{8}$ between $\widetilde{\Sigma}_{\phi}$ and $\Sigma_{\phi}$. It is clear that $W^{8}$ is simply connected. Using the MayerVietoris exact sequence of the union $D^{4} \times S^{3}=\widetilde{D^{4} \times S^{3}} \cup D^{7}$, we see that

$$
H_{*}\left(\widetilde{D^{4} \times S^{3}}\right) \cong\left\{\begin{array}{cc}
\mathbb{Z} & \text { if } *=0,3,6, \\
0 & \text { otherwise. }
\end{array}\right.
$$

In a similar way, we can get homology groups of $W^{8}=D^{5} \times S^{3} \cup D^{8}$ :

$$
H_{*}\left(W^{8}\right) \cong\left\{\begin{array}{cc}
\mathbb{Z} & \text { if } *=0 \text { or } 7 \\
0 & \text { otherwise }
\end{array} \text { and } H_{*}\left(W^{8}, \Sigma_{\phi}\right) \cong 0 .\right.
$$

Thus, by the h-cobordism theorem [17], two homotopy spheres $\widetilde{\Sigma}_{\phi}$ and $\Sigma_{\phi}$ are diffeomorphic.

Theorem 2. The generators $A, B$ and $\Sigma$ of $\pi_{0} S$ Diff $\left(S^{3} \times S^{3}\right)$ satisfy the relation: $A B A^{-1} B^{-1}=\Sigma$.

Proof. For the proof, we construct a spin manifold $W^{8}$ bounded by the sphere $\Sigma_{A B A^{-1} B^{-1}}$ and compute the $\mu$-invariant $\mu\left(\Sigma_{A B A^{-1} B^{-1}}\right)$ defined by Eells and Kuiper [7].

First we extend the diffeomorphisms $A$ and $B$ to diffeomorphisms of the handlebodies $D^{4} \times S^{3}$ and $S^{3} \times D^{4}$ respectively (recall formulas (5) ). These extensions we also denote by $A$ and $B$ respectively. Next we present $\Sigma_{A B A^{-1} B^{-1}}$ as the union of five manifolds:

$$
\begin{aligned}
& D^{4} \times S^{3} \cup_{A B A^{-1} B^{-1}} S^{3} \times D^{4} \\
& \simeq D^{4} \times S^{3} \cup_{A} S^{3} \times S^{3} \times I \cup_{B} S^{3} \times S^{3} \times I \cup_{A^{-1}} S^{3} \times S^{3} \times I \cup_{B^{-1}} S^{3} \times D^{4}
\end{aligned}
$$

where $A, B, A^{-1}$ and $B^{-1}$ belong to $\operatorname{Diff}\left(S^{3} \times S^{3}\right)$. Consider the manifolds $D^{8}$, $D^{5} \times S^{3}$ and $S^{3} \times D^{5}$ with boundaries presented as:

$$
\begin{aligned}
\partial\left(D^{5} \times S^{3}\right) & =S^{4} \times S^{3}=D^{4} \times S^{3} \cup D^{4} \times S^{3}, \\
\partial\left(S^{3} \times D^{5}\right) & =S^{3} \times S^{4}=S^{3} \times D^{4} \cup S^{3} \times D^{4}, \\
\partial\left(D^{8}\right) & =S^{7}=D^{4} \times S^{3} \cup S^{3} \times S^{3} \times I \cup S^{3} \times D^{4} .
\end{aligned}
$$

Using the extension diffeomorphisms $A$ and $B$ defined above, we now construct a manifold $W^{8}$ which will be used to compute $\mu\left(\Sigma_{A B A^{-1} B^{-1}}\right)$.

Definition 2. Define $W^{8}$ to be the manifold obtained from the union

$$
\begin{array}{lllllllll}
D^{5} \times S^{3} \cup_{A} & D^{8} \cup_{B} & D^{8} \cup_{A^{-1}} & D^{8} \cup_{B^{-1}} & S^{3} \times D^{5}
\end{array}
$$

by smoothing the corners.

Claim 2. $D^{5} \times S^{3} \cup_{A} D^{8} \simeq D^{8} \cup_{B^{-1}} S^{3} \times D^{5} \simeq D^{8}$.

Proof of claim: Evidently, the union $D^{5} \times S^{3} \cup_{D^{4} \times S^{3}} D^{8}$ is a simply connected manifold with simply connected boundary $D^{4} \times S^{3} \cup_{S^{3} \times S^{3}} S^{3} \times D^{4}$. Using the exact sequence of Mayer-Vietoris, it is easy to see that the homology groups of $D^{5} \times S^{3} \cup_{D^{4} \times S^{3}} D^{8}$ are trivial in all dimensions $>0$. Hence by the characterizations of the smooth $n$-disk $D^{n}, n \geq 6$ (see [17]) this union is diffeomorphic to the disk $D^{8}$. The same proof works in the second case. 
Thus we can write $W^{8}=D^{8} \cup_{B} D^{8} \cup_{A^{-1}} D^{8}$. Now note that $\partial\left(W^{8}\right)=M\left(f_{B}, f_{A}\right)$ where (using notation of Milnor [16]) by $M\left(f_{B}, f_{A}\right)$ we denote the boundary of the following union of three 8-disks:

$$
\left(D^{4} \times D^{4}\right)_{3} \cup_{S^{3} \times D^{4}}\left(D^{4} \times D^{4}\right)_{2} \cup_{D^{4} \times S^{3}}\left(D^{4} \times D^{4}\right)_{1} .
$$

The gluing maps are (cf. [16], §1): $\left(x_{3}, y_{3}\right) \stackrel{f_{B}}{\longleftarrow}\left(x_{2}, y_{2}\right) \stackrel{f_{A}^{-1}}{\longleftarrow}\left(x_{1}, y_{1}\right)$ where $\left(x_{i}, y_{i}\right) \in$ $\left(D^{4} \times D^{4}\right)_{i}$

$$
y_{2}=y_{1}, x_{2}=f\left(y_{1}\right)^{-1} \circ x_{1}, y_{3}=f\left(x_{2}\right) \circ y_{2}, x_{3}=x_{2}
$$

and $f_{B}=f_{A}=f: S^{3} \longrightarrow S O(3) \stackrel{i_{3}}{\hookrightarrow} S O(4)$, is defined by the formula: $f(x) \circ$ $y=x y x^{-1}$ for $x \in S^{3}$ and $y \in D^{4}$. In particular, we see that our homotopy sphere $\Sigma_{A B A^{-1} B^{-1}}$ is diffeomorphic to the manifold $M\left(f_{B}, f_{A}\right)$. Using the MayerVietoris exact sequence for the manifold $W^{8} \simeq D^{8} \cup_{B} D^{8} \cup_{A^{-1}} D^{8}$, we see that $H^{*}\left(W^{8}, \mathbb{Z}\right) \simeq 0$, for $*=1,2$ or 3 , and we can apply results of Eells and Kuiper $([7]$, $\S 10)$ to the manifold $W^{8}$ to compute the $\mu$-invariant of $M\left(f_{B}, f_{A}\right) \simeq \Sigma_{A B A^{-1} B^{-1}}$. It is shown (see 7], page 109) that

$$
\mu\left(M\left(f_{B}, f_{A}\right)\right)=\frac{B_{1}^{2}}{8(2 !)^{2}}\left(1+\frac{2}{2^{3}-1}\right)\left( \pm 2 p_{1}\left(f_{B}\right) p_{1}\left(f_{A}\right)\right)= \pm \frac{p_{1}\left(f_{B}\right) p_{1}\left(f_{A}\right)}{448}
$$

where $B_{1}=1 / 6$ is the first Bernoulli number and $p_{1}\left(f_{B}\right), p_{1}\left(f_{A}\right)$ are Pontrjagin numbers of the stable vector bundles over $S^{4}$ determined by the compositions (cf. [16], §3):

$$
S^{3} \stackrel{f_{B}}{\longrightarrow} S O(4) \stackrel{i_{4}}{\hookrightarrow} S O(5), \quad S^{3} \stackrel{f_{A}}{\longrightarrow} S O(4) \stackrel{i_{4}}{\hookrightarrow} S O(5) .
$$

We show that $p_{1}\left(f_{B}\right)=p_{1}\left(f_{A}\right)= \pm 4$. It is well known how Pontrjagin numbers depend on the characteristic maps of the stable vector bundles over the spheres : $p_{s}\left[S^{4 s}\right]= \pm a_{s} \cdot \lambda \cdot(2 s-1)$ !. Here $\lambda$ is the integer corresponding to the characteristic map: $S^{4 s-1} \longrightarrow S O$ and $a_{s}=1$ or 2 if $s$ is even or odd respectively. In our case $p_{1}\left(f_{B}\right)= \pm 2 \cdot\left[i_{4} \circ f_{B}\right]$ and to find the integer $\left[i_{4} \circ f_{B}\right]$, we need to find the homotopy class of the composition

$$
S^{3} \longrightarrow S O(3) \stackrel{i_{3}}{\hookrightarrow} S O(4) \stackrel{i_{4}}{\hookrightarrow} S O(5) .
$$

It is a standard fact (which can be deduced from Theorem IV.1.12 of [4) that the inclusion $i=i_{4} \circ i_{3}$ induces the map $i_{*}: \pi_{3}(S O(3)) \longrightarrow \pi_{3}(S O(5))$, which is multiplication by 2 . Hence $\left[i_{4} \circ f_{B}\right]=2$ and similarly $\left[i_{4} \circ f_{A}\right]=2$. Therefore $\mu\left(\Sigma_{A B A^{-1} B^{-1}}\right)=\mu\left(M\left(f_{B}, f_{A}\right)\right)= \pm \frac{16}{448}= \pm \frac{1}{28}$. For the generator $\widehat{\Sigma}$ of $\mathbb{Z}_{28}$, we have (see $\S 4$ of [7]) $\mu(\widehat{\Sigma}) \equiv \frac{-1}{2^{5} 7} \cdot 8(\bmod 1)$ or $\mu(\widehat{\Sigma})=\frac{-1}{28}$. From the theorem of Eells and Kuiper ([7], p. 103) we see (changing orientation of $\widehat{\Sigma}$ if necessary) that $\Sigma_{A B A^{-1} B^{-1}} \simeq \widehat{\Sigma}$. Since $A B A^{-1} B^{-1}=\Sigma^{k}=\iota\left(\widehat{\Sigma}^{k}\right)$, we can apply Lemma 3 to get $\widehat{\Sigma}^{k}=\varsigma \circ \iota\left(\widehat{\Sigma}^{k}\right)=\varsigma\left(A B A^{-1} B^{-1}\right)=\widehat{\Sigma}$. It shows that $k \equiv 1(\bmod 28)$ and finishes the proof.

As a corollary we get a presentation of the group $\pi_{0} S \operatorname{Diff}\left(S^{3} \times S^{3}\right)$ :

$$
\left\langle A, B, \Sigma \mid \Sigma^{28}=1, A \Sigma=\Sigma A, B \Sigma=\Sigma B, A B A^{-1} B^{-1}=\Sigma\right\rangle .
$$

Consider now the matrices:

$$
A^{\prime}=\left(\begin{array}{lll}
1 & 1 & 0 \\
0 & 1 & 0 \\
0 & 0 & 1
\end{array}\right) ; \quad B^{\prime}=\left(\begin{array}{ccc}
1 & 0 & 0 \\
0 & 1 & 1 \\
0 & 0 & 1
\end{array}\right) ; \quad \Sigma^{\prime}=\left(\begin{array}{ccc}
1 & 0 & 1 \\
0 & 1 & 0 \\
0 & 0 & 1
\end{array}\right)
$$


It is easy to verify that these matrices $A^{\prime}, B^{\prime}, \Sigma^{\prime}$ generate the group $\mathcal{H}$ and satisfy the defining relations:

$$
\left\langle A^{\prime}, B^{\prime}, \Sigma^{\prime} \mid A^{\prime} \Sigma^{\prime}=\Sigma^{\prime} A^{\prime}, B^{\prime} \Sigma^{\prime}=\Sigma^{\prime} B^{\prime}, A^{\prime} B^{\prime} A^{\prime-1} B^{\prime-1}=\Sigma^{\prime}\right\rangle .
$$

Hence the groups $\pi_{0} S$ Diff $\left(S^{3} \times S^{3}\right)$ and $\mathcal{H}_{28}$ are isomorphic.

1.4. Generators and relations. We have shown in $\S 1.2$ that the factor group $\pi_{0} \operatorname{Diff}\left(S^{3} \times S^{3}\right) / \Theta_{7}$ admits the following presentation:

$$
\begin{aligned}
& \langle Y, U, A, B| Y U Y=U Y U,(Y U Y)^{4}=1, A B=B A, \\
& \left.A Y=Y A B, A U=U A, B Y=Y B, B U=U B A^{-1}\right\rangle .
\end{aligned}
$$

Furthermore, we have shown explicitly (recall the proof of Theorem 1) that $Y U Y=$ $U Y U, A Y=Y A B, A U=U A, B Y=Y B, B U=U B A^{-1}$ as diffeomorphisms. Hence if we consider the group $\pi_{0} \operatorname{Diff}\left(S^{3} \times S^{3}\right)$ as a central extension of $\pi_{0} \operatorname{Diff}\left(S^{3} \times S^{3}\right) / \Theta_{7}$ by the group $\Theta_{7}$, we can choose $Y, U, A, B, \Sigma$ to be the generators. The defining relations will be: $Y U Y=U Y U, A Y=Y A B, A U=$ $U A, B Y=Y B, B U=U B A^{-1}, \Sigma^{28}=I d, \Sigma \leftrightarrows Y, U, A, B,(Y U Y)^{4}=\Sigma^{m}$, and $A B A^{-1} B^{-1}=\Sigma^{n}$ for some $m, n \in \mathbb{Z}(n=1$ by Theorem 2$)$. The symbol $\leftrightarrows$ means that the element on the left commutes with any element on the right.

Claim 3. The following identities are valid in the group $\pi_{0} \operatorname{Diff}\left(S^{3} \times S^{3}\right)$ :

$$
\left(B^{-1} Y U Y\right)^{4}=I d, \quad Y U Y B^{-1}=A^{-1} \Sigma Y U Y, \quad Y U Y A^{-1}=B Y U Y .
$$

Proof. We know that $\left(B^{-1} Y U Y\right)^{4}=I d$ (Claim 1). Using the identities $A B=$ $B A \Sigma, A Y=Y A B, U B=B A U$, we find that $Y U Y A^{-1}=Y U A^{-1} B \Sigma Y=$ $Y A^{-1} U B \Sigma Y=Y A^{-1} B A \Sigma U Y=Y B U Y=B Y U Y$. Similarly, $Y U Y B^{-1}=$ $Y U B^{-1} Y=Y A^{-1} B^{-1} U Y=A^{-1} B \Sigma B^{-1} Y U Y=A^{-1} \Sigma Y U Y$.

Now it is easy to see that $m=-1$. Indeed,

$$
\begin{aligned}
I d & =\left(B^{-1} Y U Y\right)^{2} B^{-1} Y U Y B^{-1} Y U Y=\left(B^{-1} Y U Y\right)^{2} B^{-1} A^{-1} \Sigma(Y U Y)^{2} \\
& =B^{-1} Y U Y B^{-1} Y U Y A^{-1} B^{-1}(Y U Y)^{2}=B^{-1} Y U Y B^{-1} B Y U Y B^{-1}(Y U Y)^{2} \\
& =B^{-1} Y U Y A^{-1} \Sigma(Y U Y)^{3}=B^{-1} B \Sigma(Y U Y)^{4}=\Sigma(Y U Y)^{4} .
\end{aligned}
$$

Hence $(Y U Y)^{4}=\Sigma^{-1}$.

Let us now collect all the information we have obtained so far and state the main theorem of this paper.

Theorem 3. The mapping class group of $S^{3} \times S^{3}$ admits the following presentation:

$$
\left\langle\begin{array}{cc|ccc}
Y & U & Y U Y=U Y U, & (Y U Y)^{4}=\Sigma^{-1}, & \Sigma \leftrightarrows Y, U, A, B \\
A & B & B U=U B A^{-1}, & A Y=Y A B, & A B=B A \Sigma, \\
\Sigma & & B Y=Y B, & A U=U A &
\end{array}\right\rangle
$$

with $Y=(y, 0), U=(u, 0), A=(a, 0), B=(b, 0), \Sigma=(i d, 1) \in \Gamma^{J} \times \mathbb{Z}_{28}$.

Remark. It is well known that the mapping class group of the 2-torus is generated by two Dehn twists. B. Wajnryb [20] has shown that for an orientable surface $S_{g}^{2}$ of genus $g \geq 2$, the mapping class group can be generated by two elements that are not Dehn twists in general. It follows from Theorem 3 and work of Y. Choie (see [6], Theorem 2.1) that $\pi_{0} \operatorname{Diff}\left(S^{3} \times S^{3}\right)$ can also be generated by two elements. 


\section{Cohomology of the Jacobi group $\Gamma^{J}$}

It is usually difficult to obtain information about a group having just generators and defining relations. The aim of this part is to give an alternative description of the mapping class group $\pi_{0} \operatorname{Diff}\left(S^{3} \times S^{3}\right)$ using the cohomology theory of groups. Since $\pi_{0} \operatorname{Diff}\left(S^{3} \times S^{3}\right)$ is a central extension of the Jacobi group $\Gamma^{J}$ by $\mathbb{Z}_{28}$, it is natural to ask what element of the group $H^{2}\left(\Gamma^{J}, \mathbb{Z}_{28}\right)$ corresponds to this extension. First we classify all central extensions of $S L_{2}(\mathbb{Z})$ by $\mathbb{Z}$ and determine a 2-cocycle that generates $H^{2}\left(S L_{2}(\mathbb{Z})\right)$. In the second section we show that $\Gamma^{J}$ is isomorphic to an amalgamated product. Using a Mayer-Vietoris exact sequence of this amalgamated product, we compute the cohomology groups of $\Gamma^{J}$. Finally, we specify elements of $H^{2}\left(\Gamma^{J}, \mathbb{Z}_{28}\right)$ and $H^{2}\left(S L_{2}(\mathbb{Z}), \mathbb{Z}_{28}\right)$ that correspond to the mapping class group $\pi_{0} \operatorname{Diff}\left(S^{3} \times S^{3}\right)$.

2.1. Central extensions of $S L_{2}(\mathbb{Z})$ and $\mathbb{Z} \oplus \mathbb{Z}$. There are several ways to find cohomology groups of $S L_{2}(\mathbb{Z})$ with trivial $\mathbb{Z}$-coefficients. All of these groups are, of course, well known. The reason why we make some calculations here is that, in the next section, these calculations will be generalized to find the group $H^{2}\left(\Gamma^{J}\right)$ and its generators.

Let $A$ be a fixed $G$-module. Consider a central extension

$$
0 \longrightarrow A \longrightarrow E \longrightarrow G \longrightarrow 0
$$

of $G$ by $A$. The equivalence classes of such extensions are in 1-1 correspondence with the elements of the second cohomology group $H^{2}(G, A)$. If $A=\mathbb{Z}$ is a trivial $G$ module, we will denote this cohomology group by $H^{2}(G)$ omitting the coefficients. Recall that $S L_{2}(\mathbb{Z}) \cong \mathbb{Z}_{4} *_{\mathbb{Z}_{2}} \mathbb{Z}_{6}$ and we can write a Mayer-Vietoris exact sequence:

$$
\rightarrow H^{n}\left(S L_{2}(\mathbb{Z})\right) \rightarrow H^{n}\left(\mathbb{Z}_{4}\right) \oplus H^{n}\left(\mathbb{Z}_{6}\right) \rightarrow H^{n}\left(\mathbb{Z}_{2}\right) \rightarrow H^{n+1}\left(S L_{2}(\mathbb{Z})\right) \rightarrow
$$

Cohomology groups of $\mathbb{Z}_{m}$ are known: $H^{2 k}\left(\mathbb{Z}_{m}\right) \cong \mathbb{Z}_{m}, H^{2 k-1}\left(\mathbb{Z}_{m}\right) \cong 0$ for $k \geq$ 1. Hence $H^{1}\left(S L_{2}(\mathbb{Z})\right) \cong 0$. We also have the following fragments:

$$
0 \rightarrow H^{2 n}\left(S L_{2}(\mathbb{Z})\right) \rightarrow H^{2 n}\left(\mathbb{Z}_{4}\right) \oplus H^{2 n}\left(\mathbb{Z}_{6}\right) \stackrel{j^{*}}{\rightarrow} H^{2 n}\left(\mathbb{Z}_{2}\right) \rightarrow H^{2 n+1}\left(S L_{2}(\mathbb{Z})\right) \rightarrow 0
$$

with $j^{*}=i_{4}^{*}+i_{6}^{*}$ and $i_{k}: \mathbb{Z}_{2} \longrightarrow \mathbb{Z}_{k}$ multiplication by $k / 2$. If we denote by $t, z$ and $z^{\prime}$ generators of groups $H^{2}\left(\mathbb{Z}_{2}\right), H^{2}\left(\mathbb{Z}_{4}\right)$ and $H^{2}\left(\mathbb{Z}_{6}\right)$ respectively, then it is easy to see that $i_{4}^{*}(z)=i_{6}^{*}\left(z^{\prime}\right)=t$, i.e., $j^{*}(n, m)=n+m$. Hence $\operatorname{Im}\left(j^{*}\right)=H^{2}\left(\mathbb{Z}_{2}\right)$, and $\operatorname{Ker}\left(j^{*}\right)$ is generated by the element $(1,5) \in \mathbb{Z}_{4} \oplus \mathbb{Z}_{6}$ and is isomorphic to $\mathbb{Z}_{12}$. Thus $H^{3}\left(S L_{2}(\mathbb{Z})\right) \cong 0$ and $H^{2}\left(S L_{2}(\mathbb{Z})\right) \cong \mathbb{Z}_{12}$. It follows from the properties of the norm map $\left(\left[\underline{5}\right.\right.$, Ch. III) that $H^{2 k-1}\left(S L_{2}(\mathbb{Z})\right) \cong 0$ and $H^{2 k}\left(S L_{2}(\mathbb{Z})\right) \cong \mathbb{Z}_{12}$ for any $k \geq 1$.

Now we write down an explicit function $f: S L_{2}(\mathbb{Z}) \times S L_{2}(\mathbb{Z}) \longrightarrow \mathbb{Z}$ that generates the group $H^{2}\left(S L_{2}(\mathbb{Z})\right)$. This function will be defined in terms of a generator of the group $H^{2}\left(\mathbb{Z}_{12}\right)$, which we describe first. Consider the group $\mathbb{Z}_{m}$ as the multiplicative group on elements $\left\{z^{1}, \ldots, z^{p}, \ldots, z^{m-1}, i d\right\}$ and define a function $f_{m}: \mathbb{Z}_{m} \times \mathbb{Z}_{m} \longrightarrow \mathbb{Z}$ by the formula:

\section{Definition 3.}

$$
f_{m}\left(z^{p}, z^{q}\right) \stackrel{\text { def }}{=}\left\{\begin{array}{lll}
1 & \text { if } & \bar{p}+\bar{q} \geq m \\
0 & \text { if } \quad \bar{p}+\bar{q}<m
\end{array}\right.
$$

where $\bar{p}, \bar{q} \in\{0,1,2, \ldots, m-1\}$ and $\bar{p} \equiv p(\bmod m), \bar{q} \equiv q(\bmod m)$.

Lemma 4. The function $f_{m}$ is a generator of the group $H^{2}\left(\mathbb{Z}_{m}\right) \cong \mathbb{Z}_{m}$. 
Proof. Equality $f_{m}\left(z^{p}, z^{q}\right)+f_{m}\left(z^{p+q}, z^{r}\right)=f_{m}\left(z^{q}, z^{r}\right)+f_{m}\left(z^{p}, z^{q+r}\right)$ shows that $f_{m}$ is a 2-cocycle. Verification of this equality is straightforward and is left as an exercise. Let $\langle c\rangle$ and $\left\langle z \mid z^{m}=i d\right\rangle$ be presentations of the groups $\mathbb{Z}$ and $\mathbb{Z}_{m}$ respectively. Then a central extension of $\mathbb{Z}_{m}$ by $\mathbb{Z}$ will have a presentation: $\left\langle Z, C \mid Z C=C Z, Z^{m}=C^{k}\right\rangle$ with $Z=(z, 0), C=(i d, 1) \in \mathbb{Z}_{m} \times \mathbb{Z}$ and some $k \in \mathbb{Z}$. It follows from formula (8) that $k=1$ for the function $f_{m}$, and that the cocycle $t \cdot f_{m}$ defines the extension with $k=t$. We denote this extension by $E_{t}$. If $m \nmid s-t$, then cocycles $s \cdot f_{m}$ and $t \cdot f_{m}$ define nonequivalent extensions. Indeed, suppose that $E_{s}$ and $E_{t}$ are equivalent. Then there exists an isomorphism $\iota: E_{s} \longrightarrow E_{t}$ that makes the following diagram commute:

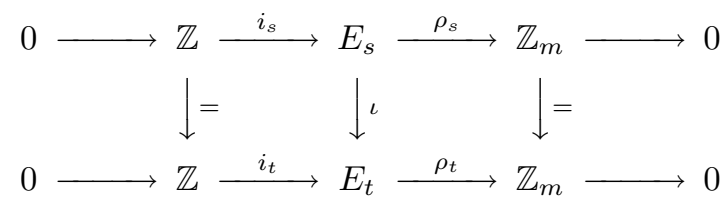

Using the above presentation of groups $E_{s}$ and $E_{t}$, one can see from this diagram that $s-t=l m$ for some $l \in \mathbb{Z}$. Hence $f_{m}$ is a generator of the group $H^{2}\left(\mathbb{Z}_{m}\right)$.

Now consider the canonical projection $a b: S L_{2}(\mathbb{Z}) \longrightarrow\left(S L_{2}(\mathbb{Z})_{a b} \cong \mathbb{Z}_{12}\right.$ and define a function $f: S L_{2}(\mathbb{Z}) \times S L_{2}(\mathbb{Z}) \longrightarrow \mathbb{Z}$ as follows.

Definition 4. $f(M, N) \stackrel{\text { def }}{=} f_{12}\left(M_{a b}, N_{a b}\right)$ where $M_{a b}=a b(M) \in \mathbb{Z}_{12}$.

Lemma 5. The function $f(M, N)=f_{12}\left(M_{a b}, N_{a b}\right)$ is a generator of the group $H^{2}\left(S L_{2}(\mathbb{Z})\right)$

Proof. Since $(M \cdot N)_{a b}=M_{a b} \cdot N_{a b}$, it follows from the previous lemma that $f$ is a 2-cocycle. Let $\langle c\rangle$ and $\left\langle y, u \mid y u y=u y u,(y u y)^{4}=i d\right\rangle$ be presentations of the groups $\mathbb{Z}$ and $S L_{2}(\mathbb{Z})$. Then a central extension of $S L_{2}(\mathbb{Z})$ by $\mathbb{Z}$ has a presentation:

$$
\left\langle Y, U, C \mid Y U Y=U Y U C^{a},(Y U Y)^{4}=C^{b}, C Y=Y C, C U=U C\right\rangle
$$

with $Y=(y, 0), U=(u, 0), C=(i d, 1)$ and some $a, b \in \mathbb{Z}$. Using group law, $(g, k) \cdot(h, l)=(g h, k+l+f(g, h))$, one can easily find that function $f$ defines the extension with $a=0$ and $b=1$. The cocycle $t \cdot f$ defines the extension with $a=0$ and $b=t$. As in the proof of Lemma 4, one can show that if $12 \nmid s-t$, then cocycles $s \cdot f$ and $t \cdot f$ define nonequivalent extensions. Therefore function $f$ is indeed a generator of $H^{2}\left(S L_{2}(\mathbb{Z})\right)$.

Since the 2-torus is an Eilenberg-MacLane complex of type $\left(\mathbb{Z}^{2}, 1\right)$, we have $H^{2}\left(\mathbb{Z}^{2}\right) \cong \mathbb{Z}$. We can write an explicit function $g: \mathbb{Z}^{2} \times \mathbb{Z}^{2} \longrightarrow \mathbb{Z}$ that generates this cohomology group. For instance, take two vectors $v_{1}=(a, b), v_{2}=(s, t) \in \mathbb{Z}^{2}$ and consider $g: \mathbb{Z}^{2} \times \mathbb{Z}^{2} \longrightarrow \mathbb{Z}$ defined by the formula: $g\left(v_{1}, v_{2}\right) \stackrel{\text { def }}{=}$ at. One can check that $g$ is a 2-cocycle that defines the following extension:

$$
\left\langle A, B, C \mid A C=C A, B C=C B, A B A^{-1} B^{-1}=C\right\rangle
$$

with $A=((1,0) ; 0), B=((0,1) ; 0), C=((0,0) ; 1) \in \mathbb{Z}^{2} \times \mathbb{Z}$. It can be shown (as we just did above) that any function that defines extension (9) is a generator of the group $H^{2}\left(\mathbb{Z}^{2}\right)$.

Remark. Consider another function $\varphi: \mathbb{Z}^{2} \times \mathbb{Z}^{2} \longrightarrow \mathbb{Z}$ defined as follows. For $v=(\lambda, \mu)$ and $w=\left(\lambda^{\prime}, \mu^{\prime}\right), \varphi(v, w) \stackrel{\text { def }}{=}\left|\begin{array}{cc}\lambda & \mu \\ \lambda^{\prime} & \mu^{\prime}\end{array}\right|=\lambda \mu^{\prime}-\lambda^{\prime} \mu$. It can be easily 
verified that $\varphi$ is a cocycle cohomologous to $2 g$. Note that $\varphi$ defines the extension that is isomorphic to the Heisenberg group $H(\mathbb{Z})$. This latter one is $\mathbb{Z} \oplus \mathbb{Z} \oplus \mathbb{Z}$ as a set and with multiplication:

$$
(a, b, c) \cdot\left(a^{\prime}, b^{\prime}, c^{\prime}\right) \stackrel{\text { def }}{=}\left(a+a^{\prime}, b+b^{\prime}, c+c^{\prime}+a \cdot b^{\prime}-b \cdot a^{\prime}\right) .
$$

Here one can consider the so-called (real) Jacobi group $G^{J}(\mathbb{Z})$ (cf. 1]) defined as the semidirect product of $S L_{2}(\mathbb{Z})$ and $H(\mathbb{Z}): G^{J}(\mathbb{Z})=S L_{2}(\mathbb{Z}) \ltimes H(\mathbb{Z})$.

2.2. Group $\Gamma^{J}$ as an amalgamated product. In this paragraph we will compute the cohomology groups of $\Gamma^{J}$. First we present $\Gamma^{J}$ as an amalgamated product and then use a Mayer-Vietoris exact sequence of this product to find $H^{2}\left(\Gamma^{J}\right)$.

Consider for $m=2,4,6$ the cyclic groups $\mathbb{Z}_{m}$ generated by the matrices

$$
\left(\begin{array}{cc}
-1 & 0 \\
0 & -1
\end{array}\right), \quad\left(\begin{array}{cc}
0 & -1 \\
1 & 0
\end{array}\right), \quad\left(\begin{array}{cc}
0 & 1 \\
-1 & 1
\end{array}\right)
$$

respectively. These are subgroups of $S L_{2}(\mathbb{Z})$ and they act on the elements of $\mathbb{Z} \oplus \mathbb{Z}$ in the same natural way as $S L_{2}(\mathbb{Z})$ does. With respect to this action, we define the semidirect products $G_{m} \stackrel{\text { def }}{=} \mathbb{Z}_{m} \ltimes \mathbb{Z}^{2}$.

Lemma 6. Group $\Gamma^{J}$ is isomorphic to the amalgamated product $G_{4} *_{G_{2}} G_{6}$.

Proof. First we give presentations of groups $G_{m}$ with generators and defining relations. Denote the generators of $\mathbb{Z}_{2}, \mathbb{Z}_{4}, \mathbb{Z}_{6}$ by $\alpha, \beta, \gamma$ and elements $(i d,(1,0))$, $(i d,(0,1))$ of the group $G_{m}$ by $A$ and $B$ respectively.

Note. To avoid cumbersome notation, we use the same letters $A$ and $B$ to denote elements of different groups. We hope it will not cause any confusion.

Using the group law $(M, X) \cdot\left(M^{\prime}, X^{\prime}\right)=\left(M M^{\prime}, X M^{\prime}+X\right)$, one can easily show (cf. proof of Lemma 1 above) that $A \alpha=\alpha A^{-1}, B \alpha=\alpha B^{-1}, B \beta=\beta A^{-1}, A \beta=$ $\beta B, A \gamma=\gamma B$, and $B \gamma=A \gamma A^{-1}$. Hence we get presentations:

$$
\begin{aligned}
G_{2} \simeq & \left\langle A, B, \alpha \mid A B=B A, \alpha^{2}=i d, B \alpha=\alpha B^{-1}, A \alpha=\alpha A^{-1}\right\rangle \\
& \Rightarrow\left(G_{2}\right)_{a b} \simeq \mathbb{Z}_{2} \oplus \mathbb{Z}_{2} \oplus \mathbb{Z}_{2}, \\
G_{4} \simeq & \left\langle A, B, \beta \mid A B=B A, \beta^{4}=i d, B \beta=\beta A^{-1}, A \beta=\beta B\right\rangle \\
& \Rightarrow\left(G_{4}\right)_{a b} \simeq \mathbb{Z}_{4} \oplus \mathbb{Z}_{2}, \\
G_{6} \simeq & \left\langle A, B, \gamma \mid A B=B A, \gamma^{6}=i d, B \gamma=A \gamma A^{-1}, A \gamma=\gamma B\right\rangle \\
& \Rightarrow\left(G_{6}\right)_{a b} \simeq \mathbb{Z}_{6} .
\end{aligned}
$$

Next define maps $\iota_{4}: G_{2} \longrightarrow G_{4}$ and $\iota_{6}: G_{2} \longrightarrow G_{6}$ by the formulas: $\iota_{4}(A)=$ $A, \iota_{4}(B)=B, \iota_{4}(\alpha)=\beta^{2}$ and $\iota_{6}(A)=A, \iota_{6}(B)=B, \iota_{6}(\alpha)=\gamma^{3}$. Map $\iota_{4}$ induces the commutative diagram

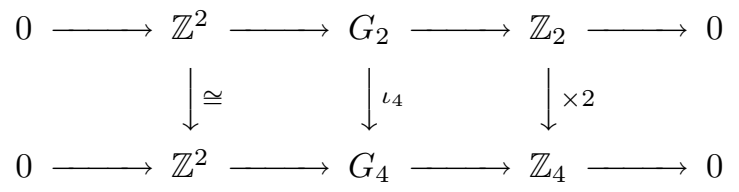

from which it follows that $\iota_{4}$ is a monomorphism. Similarly, one proves that $\iota_{6}$ is a monomorphism. Since $\alpha$ is identified with $\beta^{2}$ and $\gamma^{3}$, we obtain the following 
presentation of $G_{4} *_{G_{2}} G_{6}$ with generators and defining relations:

$$
\left\langle\begin{array}{cc|ccc}
\beta & \gamma & A B=B A, & A \beta=\beta B, & A \gamma=\gamma B, \quad B \gamma=A \gamma A^{-1} \\
A & B & B \beta=\beta A^{-1}, & \beta^{2}=\gamma^{3}, \quad \beta^{4}=i d
\end{array}\right\rangle .
$$

Consider now two elements: $U \stackrel{\text { def }}{=} \beta \gamma^{-1}$ and $Y \stackrel{\text { def }}{=} \gamma^{2} \beta^{-1}$. Obviously, $Y U=\gamma$ and $U Y U=\beta$. One can easily show that the above presentation of the group $G_{4} *_{G_{2}} G_{6}$ is equivalent to the presentation of the Jacobi group $\Gamma^{J}$ obtained in Lemma 1.

To find the cohomology of $G_{m}$, we will use the Lyndon-Hochschild-Serre (LHS) spectral sequence (see [11] or 9], $\S 7.2$ ) of the split extension that defines $G_{m}$ : $E_{2}^{p, q}=H^{p}\left(\mathbb{Z}_{m}, H^{q}\left(\mathbb{Z}^{2}, \mathbb{Z}\right)\right) \Rightarrow H^{p+q}\left(G_{m}, \mathbb{Z}\right)$. We need to know how $\mathbb{Z}_{m}$ acts on $H^{q}\left(\mathbb{Z}^{2}\right)$. Suppose $f \in H^{q}\left(\mathbb{Z}^{2}\right)$, that is, $f: \mathbb{Z}^{2} \times \cdots \times \mathbb{Z}^{2} \longrightarrow \mathbb{Z}$. Then we have $\mathcal{M} \circ f\left(\sigma_{1}, \ldots, \sigma_{q}\right)=f\left(\mathcal{M}^{-1} \sigma_{1} \mathcal{M}, \ldots, \mathcal{M}^{-1} \sigma_{q} \mathcal{M}\right)$ (since $\mathbb{Z}$ is a trivial $\mathbb{Z}_{m}$-module) where

$$
\sigma_{i}=\left(\left(\begin{array}{ll}
1 & 0 \\
0 & 1
\end{array}\right) ;\left(x_{i}, y_{i}\right)\right) \quad \text { and } \quad \mathcal{M}=\left(\left(\begin{array}{ll}
a & b \\
c & d
\end{array}\right) ;(0,0)\right)
$$

are the corresponding elements of the group $G_{m}$ (cf. [11], p. 117). If we denote by $M$ the matrix of the element $\mathcal{M}$ and by $\sigma_{i}$ the vector of the element $\sigma_{i}$, we find that $M \circ f\left(\sigma_{i}, \ldots, \sigma_{q}\right)=f\left(\sigma_{i} \cdot M, \ldots, \sigma_{q} \cdot M\right)$ where on the right we mean multiplication of the row vector by a matrix. Groups $H^{q}\left(\mathbb{Z}^{2}\right)$ are nonzero only for $q=0,1,2$.

It is easy to verify that $\mathbb{Z}_{m}$ acts trivially on the groups $H^{0}\left(\mathbb{Z}^{2}\right) \cong \mathbb{Z}$ and $H^{2}\left(\mathbb{Z}^{2}\right) \cong$ $\mathbb{Z}$. If we denote by $(n, k)$ an element of $H^{1}\left(\mathbb{Z}^{2}\right) \cong \mathbb{Z} \oplus \mathbb{Z}$, it can be shown that $M \circ(n, k)=(n, k) \cdot M^{T}$.

Since groups $H^{*}\left(\mathbb{Z}_{m}\right)$ are well known, we only need to compute $H^{*}\left(\mathbb{Z}_{m}, \mathbb{Z}^{2}\right)$ with respect to the action described above. $H^{*}\left(\mathbb{Z}_{m}, \mathbb{Z}^{2}\right)$ can be found using the norm map $\bar{N}: M_{G} \longrightarrow M^{G}$ for group $G=\mathbb{Z}_{m}$ and the module $M=\mathbb{Z}^{2}$, since $H^{2 k}(G, M) \cong \operatorname{coker} \bar{N}$ and $H^{2 k-1}(G, M) \cong \operatorname{ker} \bar{N}$ for any $k \geq 1$ (see [5], Ch. III).

1) For $\mathbb{Z}_{2}$ we have $M_{G} \cong \mathbb{Z}_{2} \oplus \mathbb{Z}_{2}$ with the generators $(1,0),(0,1)$. It is obvious that $M^{G} \cong 0$ and we get:

$$
H^{2 k}\left(\mathbb{Z}_{2}, \mathbb{Z}^{2}\right) \cong 0, \quad H^{2 k+1}\left(\mathbb{Z}_{2}, \mathbb{Z}^{2}\right) \cong \mathbb{Z}_{2} \oplus \mathbb{Z}_{2} \quad \text { for any } k \geq 0 .
$$

2) For $\mathbb{Z}_{4}$ we have $M_{G} \cong \mathbb{Z}_{2}$ with the generator $(1,0)$. Here $M^{G} \cong 0$ also, and we get: $H^{2 k}\left(\mathbb{Z}_{4}, \mathbb{Z}^{2}\right) \cong 0, H^{2 k+1}\left(\mathbb{Z}_{4}, \mathbb{Z}^{2}\right) \cong \mathbb{Z}_{2}$ for any $k \geq 0$.

3 ) In the case of $\mathbb{Z}_{6}, M_{G} \cong M^{G} \cong 0$ and $H^{k}\left(\mathbb{Z}_{6}, \mathbb{Z}^{2}\right) \cong 0$ for any $k \geq 0$.

Lemma 7. For any $k \geq 0$ and $m \in\{2,4,6\}, H^{2 k+1}\left(G_{m}\right) \cong 0$. Furthermore,

$$
\begin{aligned}
& H^{2}\left(G_{2}\right) \cong H^{0}\left(\mathbb{Z}_{2}\right) \oplus H^{1}\left(\mathbb{Z}_{2}, \mathbb{Z} \oplus \mathbb{Z}\right) \oplus H^{2}\left(\mathbb{Z}_{2}\right) \cong \mathbb{Z} \oplus\left(\mathbb{Z}_{2} \oplus \mathbb{Z}_{2}\right) \oplus \mathbb{Z}_{2}, \\
& H^{2}\left(G_{4}\right) \cong H^{0}\left(\mathbb{Z}_{4}\right) \oplus H^{1}\left(\mathbb{Z}_{4}, \mathbb{Z} \oplus \mathbb{Z}\right) \oplus H^{2}\left(\mathbb{Z}_{4}\right) \cong \mathbb{Z} \oplus \mathbb{Z}_{2} \oplus \mathbb{Z}_{4}, \\
& H^{2}\left(G_{6}\right) \cong H^{0}\left(\mathbb{Z}_{6}\right) \oplus H^{2}\left(\mathbb{Z}_{6}\right) \cong \mathbb{Z} \oplus \mathbb{Z}_{6}
\end{aligned}
$$

Proof. We prove this lemma only for $m=2$. In the other cases, the proof is similar. Consider the first quadrant of the LHS spectral sequence $E_{2}^{p, q}=H^{p}\left(\mathbb{Z}_{2}, H^{q}\left(\mathbb{Z}^{2}\right)\right)$, 
for $p, q \geq 0$ :

\begin{tabular}{ccccccc}
$\uparrow \vdots$ & $\vdots$ & $\vdots$ & $\vdots$ & $\vdots$ & $\vdots$ & \\
0 & 0 & 0 & 0 & 0 & 0 & $\cdots$ \\
$\mathbb{Z}$ & 0 & $\mathbb{Z}_{2}$ & 0 & $\mathbb{Z}_{2}$ & 0 & $\cdots$ \\
0 & $\mathbb{Z}_{2}^{2}$ & 0 & $\mathbb{Z}_{2}^{2}$ & 0 & $\mathbb{Z}_{2}^{2}$ & $\cdots$ \\
$\mathbb{Z}$ & 0 & $\mathbb{Z}_{2}$ & 0 & $\mathbb{Z}_{2}$ & 0 & $\cdots$ \\
\hline
\end{tabular}

From this $E_{2}$-term, we see that $E_{2}^{*, *} \cong E_{3}^{*, *} \cong \ldots \cong E_{\infty}^{*, *}$. Hence for any $k \geq 0$, $H^{2 k+1}\left(G_{2}\right) \cong 0$. Since $H_{1}\left(G_{2}\right) \cong\left(G_{2}\right)_{a b} \cong \mathbb{Z}_{2} \oplus \mathbb{Z}_{2} \oplus \mathbb{Z}_{2}$, by the Universal Coefficient Theorem, $H^{2}\left(G_{2}\right) \cong \operatorname{Hom}\left(H_{2}\left(G_{2}\right), \mathbb{Z}\right) \oplus \operatorname{Ext}\left(H_{1}\left(G_{2}\right), \mathbb{Z}\right) \cong H_{o m}\left(H_{2}\left(G_{2}\right), \mathbb{Z}\right) \oplus \mathbb{Z}_{2}^{3}$, and therefore $H^{2}\left(G_{2}\right) \cong H^{0}\left(\mathbb{Z}_{2}\right) \oplus H^{1}\left(\mathbb{Z}_{2}, \mathbb{Z}^{2}\right) \oplus H^{2}\left(\mathbb{Z}_{2}\right) \cong \mathbb{Z} \oplus\left(\mathbb{Z}_{2} \oplus \mathbb{Z}_{2}\right) \oplus \mathbb{Z}_{2}$.

Theorem 4. $\Gamma^{J}$ has the following homology and cohomology groups:

$$
\begin{aligned}
& H_{1}\left(\Gamma^{J}\right) \cong \mathbb{Z}_{12}, \\
& H_{2}\left(\Gamma^{J}\right) \cong \mathbb{Z} \oplus \mathbb{Z}_{2}, \\
& H^{1}\left(\Gamma^{J}\right) \cong 0 \\
& H^{2}\left(\Gamma^{J}\right) \cong \mathbb{Z} \oplus \mathbb{Z}_{12}, \\
& H^{3}\left(\Gamma^{J}\right) \cong \mathbb{Z}_{2} .
\end{aligned}
$$

Proof. First note that it follows from the presentation of the group $\Gamma^{J}$ that $H_{1}\left(\Gamma^{J}\right)$ $\cong\left(\Gamma^{J}\right)_{a b} \cong \mathbb{Z}_{12}$. To compute the cohomology groups, we use a Mayer-Vietoris exact sequence of the amalgamated product $G_{4} *_{G_{2}} G_{6}$. By the previous lemma, we get the following fragment of this exact sequence:

$$
0 \longrightarrow H^{2}\left(\Gamma^{J}\right) \longrightarrow H^{2}\left(G_{4}\right) \oplus H^{2}\left(G_{6}\right) \stackrel{j}{\longrightarrow} H^{2}\left(G_{2}\right) \longrightarrow H^{3}\left(\Gamma^{J}\right) \longrightarrow 0
$$

where $j=\iota_{4}^{*}+\iota_{6}^{*}$ and $\iota_{m}^{*}$ is induced by the inclusion $\iota_{m}: G_{2} \hookrightarrow G_{m}$ (cf. proof of Lemma 6). The commutative diagram (10) together with the functorial dependence (cf. [9], §7.2) induce the functorial map $H^{p}\left(\mathbb{Z}_{4}, H^{q}\left(\mathbb{Z}^{2}\right)\right) \longrightarrow H^{p}\left(\mathbb{Z}_{2}, H^{q}\left(\mathbb{Z}^{2}\right)\right.$ ). Hence $H^{2}\left(G_{4}\right) \stackrel{\iota_{4}^{*}}{\longrightarrow} H^{2}\left(G_{2}\right)$ is the map induced by multiplication: $\mathbb{Z}_{2} \stackrel{\times 2}{\longrightarrow} \mathbb{Z}_{4}$. If we denote by $x, y, z$ generators of the groups $H^{0}\left(\mathbb{Z}_{4}\right) \cong \mathbb{Z}, H^{1}\left(\mathbb{Z}_{4}, \mathbb{Z}^{2}\right) \cong \mathbb{Z}_{2}, H^{2}\left(\mathbb{Z}_{4}\right) \cong$ $\mathbb{Z}_{4}$ respectively, then we see that $\iota_{4}^{*}(x, y, z)=\left(x^{\prime}, y^{\prime}, 0, z^{\prime}\right)$ where $x^{\prime}, y^{\prime}, z^{\prime}$ are generators of the groups $\mathbb{Z} \cong H^{0}\left(\mathbb{Z}_{2}\right), \mathbb{Z}_{2} \subset H^{1}\left(\mathbb{Z}_{2}, \mathbb{Z}^{2}\right), \mathbb{Z}_{2} \cong H^{2}\left(\mathbb{Z}_{2}\right)$. Similarly, one can show that $\iota_{6}^{*}(s, t)=\left(x^{\prime}, 0,0, z^{\prime}\right)$ where by $s$ and $t$ we denote the generators of the groups $H^{0}\left(\mathbb{Z}_{6}\right)$ and $H^{2}\left(\mathbb{Z}_{6}\right)$. Hence $H^{3}\left(\Gamma^{J}\right) \cong \operatorname{Coker}(j) \cong \mathbb{Z}_{2}$ and $H^{2}\left(\Gamma^{J}\right) \cong \operatorname{Ker}(j) \cong \mathbb{Z} \oplus \mathbb{Z}_{12}$. It is clear that $H^{1}\left(\Gamma^{J}\right) \cong 0$. We can assume that $H_{2}\left(\Gamma^{J}\right) \cong \mathbb{Z}^{n_{2}} \oplus$ Tor $_{2}$ and $H_{3}\left(\Gamma^{J}\right) \cong \mathbb{Z}^{n_{3}} \oplus$ Tor $_{3}$ where by Tor $_{k}$ we denote the elements of finite order. Then by the Universal Coefficient Theorem, $\mathbb{Z} \oplus \mathbb{Z}_{12} \cong H^{2}\left(\Gamma^{J}\right) \cong \operatorname{Ext}\left(\mathbb{Z}_{12}, \mathbb{Z}\right) \oplus \operatorname{Hom}\left(\mathbb{Z}^{n_{2}} \oplus T\right.$ or $\left.2, \mathbb{Z}\right) \cong \mathbb{Z}_{12} \oplus \mathbb{Z}^{n_{2}}$, that is, $n_{2}=1$. Similarly, we have $\mathbb{Z}_{2} \cong H^{3}\left(\Gamma^{J}\right) \cong \operatorname{Ext}\left(\mathbb{Z} \oplus \operatorname{Tor}_{2}, \mathbb{Z}\right) \oplus \operatorname{Hom}\left(\mathbb{Z}^{n_{3}} \oplus \operatorname{Tor}_{3}, \mathbb{Z}\right) \cong$ $\operatorname{Ext}\left(\operatorname{Tor}_{2}, \mathbb{Z}\right) \oplus \mathbb{Z}^{n_{3}}$. Therefore, $n_{3}=0$ and Tor $_{2} \cong \mathbb{Z}_{2}$ as required.

Remark. To find the other cohomology groups of $\Gamma^{J}$, one could use the LHS spectral sequence of the defining extension of $\Gamma^{J}: H^{p}\left(S L_{2}(\mathbb{Z}), H^{q}\left(\mathbb{Z}^{2}\right)\right) \Rightarrow H^{p+q}\left(\Gamma^{J}\right)$. It can be seen that $H^{*}\left(S L_{2}(\mathbb{Z}), H^{2}\left(\mathbb{Z}^{2}\right)\right) \cong H^{*}\left(S L_{2}(\mathbb{Z}), H^{0}\left(\mathbb{Z}^{2}\right)\right) \cong H^{*}\left(\mathbb{Z}_{12}\right)$ and $H^{2 k-1}\left(S L_{2}(\mathbb{Z}), H^{1}\left(\mathbb{Z}^{2}\right)\right) \cong 0$ for any $k \geq 1$. The previous theorem implies that $d_{2}^{0,2}$ of this spectral sequence is the zero map. Using the cup product, we 
deduce that $d_{2}^{2,2}$ is zero; hence $d_{2}^{4,2}$ is zero and so on. Applying Proposition 7.3.2 of [9] and Theorem 4 of [11], we see that

$$
H^{2 k+1}\left(\Gamma^{J}\right) \cong \mathbb{Z}_{2} \quad \text { and } \quad H^{2 k+2}\left(\Gamma^{J}\right) \cong \mathbb{Z}_{12} \oplus \mathbb{Z}_{12} \quad \text { for any } k \geq 1 .
$$

Theorem 5. The split extension $0 \longrightarrow \mathbb{Z}^{2} \stackrel{i}{\longrightarrow} \Gamma^{J} \stackrel{\rho}{\longrightarrow} S L_{2}(\mathbb{Z}) \longrightarrow 0$ induces the following split exact sequence (compare with the five-term exact sequence [9], §7.2):

$$
0 \longrightarrow H^{2}\left(S L_{2}(\mathbb{Z})\right) \stackrel{\rho^{*}}{\longrightarrow} H^{2}\left(\Gamma^{J}\right) \stackrel{i^{*}}{\longrightarrow} H^{2}\left(\mathbb{Z}^{2}\right) \longrightarrow 0 .
$$

Proof. Choose the presentation of $\Gamma^{J}$ found in Lemma 1. Then any central extension $E: 0 \rightarrow \mathbb{Z} \rightarrow E \stackrel{\pi}{\rightarrow} \Gamma^{J} \rightarrow 0$ has the following presentation:

$$
\left\langle\begin{array}{cc|ccc}
Y & U & Y U Y=U Y U \Sigma^{k_{0}}, & (Y U Y)^{4}=\Sigma^{k_{1}}, & A B=B A \Sigma^{k_{2}}, \\
A & B & B U=U B A^{-1} \Sigma^{k_{3}}, & A Y=Y A B \Sigma^{k_{4}}, & A U=U A \Sigma^{k_{5}}, \\
\Sigma & & B Y=Y B \Sigma^{k_{6}}, & \Sigma \leftrightarrows Y, U, A, B &
\end{array}\right\rangle
$$

with some $k_{0}, \ldots, k_{6} \in \mathbb{Z}$ and $Y=(y, 0), U=(u, 0), A=(a, 0), B=(b, 0)$ and $\Sigma=(i d, 1)$. Consider the element $\mathbf{U} \stackrel{\text { def }}{=} U \Sigma^{k_{0}}$. Then from the equality $Y U Y=U Y U \Sigma^{k_{0}}$, we get $Y \mathbf{U} Y=\mathbf{U} Y \mathbf{U}$. Instead of $(Y U Y)^{4}=\Sigma^{k_{1}}$, we get $(Y \mathbf{U} Y)^{4}=\Sigma^{k_{1}+4 k_{0}}$ and so on. Thus, by changing $U$ to $U \Sigma^{k_{0}}$, we can make $k_{0}=0$. Similarly, changing $A$ to $A \Sigma^{-k_{3}}$ and $B$ to $B \Sigma^{k_{4}}$, we can eliminate $k_{3}$ and $k_{4}$ and assume that $k_{0}=k_{3}=k_{4}=0$ in the above presentation of $E$. Now one can easily obtain the following equalities: $B Y U Y=Y U Y A^{-1} \Sigma^{2 k_{6}}, B U Y U=$ $U Y U A^{-1} \Sigma^{k_{6}-k_{5}}$. Therefore $k_{6}=-k_{5}$. Analogously, if we compare $A Y U Y$ with $A U Y U$, we find that $k_{6}=k_{5}$. Hence $k_{5}=k_{6}=0$. This means that we can choose set-theoretic crosssections of $\pi$ (i.e., functions $s_{m, n}: \Gamma^{J} \longrightarrow E$ so that $\pi \circ s_{m, n}=i d$ ) in such a way that any central extension of $\Gamma^{J}$ by $\mathbb{Z}$ has a presentation:

$$
E_{m, n} \stackrel{\text { def }}{=}\left\langle\begin{array}{cc|ccc}
Y & U & Y U Y=U Y U, & (Y U Y)^{4}=\Sigma^{n}, & A B=B A \Sigma^{m}, \\
A & B & B U=U B A^{-1}, & A Y=Y A B, & A U=U A, \\
\Sigma & & B Y=Y B, & \Sigma \leftrightarrows Y, U, A, B &
\end{array}\right\rangle
$$

and any element of $H^{2}\left(\Gamma^{J}\right)$ will be cohomologous to a cocycle defined by one of these $s_{m, n}$.

Choose a 2-cocycle $\omega_{1}$ that defines the extension $E_{1,0}$. Evidently, $i^{*}\left(\omega_{1}\right)$ defines the extension (9) of $\mathbb{Z}^{2}$ by $\mathbb{Z}$ and therefore generates $H^{2}\left(\mathbb{Z}^{2}\right)$. Hence $i^{*}$ is onto and $\omega_{1}$ generates the direct summand $\mathbb{Z}$ of $H^{2}\left(\Gamma^{J}\right)$. As for the other generator $\omega_{2}$ of $\mathbb{Z}_{12} \subset H^{2}\left(\Gamma^{J}\right)$, we can choose $\rho^{*}(f)$ (recall Def. 4 above). It can be verified that $\omega_{2}=\rho^{*}(f)$ defines the extension $E_{0,1}$ and the subgroup generated by $\omega_{2}$ is the kernel of $i^{*}$. This proves the exactness. Proof of the asserted splitting is left to the reader (cf. Proposition 7.3.2. of [9]).

2.3. On groups $H^{2}\left(\Gamma^{J}, \mathbb{Z}_{28}\right)$ and $\pi_{0} \operatorname{Diff}\left(S^{3} \times S^{3}\right)$. In this paragraph we determine elements of the groups $H^{2}\left(\Gamma^{J}, \mathbb{Z}_{28}\right)$ and $H^{2}\left(S L_{2}(\mathbb{Z}), \mathbb{Z}_{28}\right)$ that correspond to the mapping class group of $S^{3} \times S^{3}$.

Consider a short exact sequence $0 \longrightarrow A \stackrel{\mu}{\longrightarrow} B \stackrel{\nu}{\longrightarrow} C \longrightarrow 0$ of $G$-modules. Then there is a natural map $\delta: H^{n}(G, C) \longrightarrow H^{n+1}(G, A)$ such that the sequence

$$
H^{n}(G, A) \stackrel{\mu_{n}}{\longrightarrow} H^{n}(G, B) \stackrel{\nu_{n}}{\longrightarrow} H^{n}(G, C) \stackrel{\delta}{\longrightarrow} H^{n+1}(G, A)
$$


is exact. Therefore, the short exact sequence $0 \longrightarrow \mathbb{Z} \stackrel{\times 28}{\longrightarrow} \mathbb{Z} \longrightarrow \mathbb{Z}_{28} \longrightarrow 0$ of trivial $\Gamma^{J}$-modules induces the long exact sequence

$$
H^{2}\left(\Gamma^{J}\right) \stackrel{\mu_{2}}{\longrightarrow} H^{2}\left(\Gamma^{J}\right) \stackrel{\nu_{2}}{\longrightarrow} H^{2}\left(\Gamma^{J}, \mathbb{Z}_{28}\right) \stackrel{\delta}{\longrightarrow} H^{3}\left(\Gamma^{J}\right) \stackrel{\mu_{3}}{\longrightarrow} H^{3}\left(\Gamma^{J}\right) .
$$

The map $\mu_{n}$ is multiplication by 28 and we have (from Theorem 4) that $H^{2}\left(\Gamma^{J}, \mathbb{Z}_{28}\right) \cong \operatorname{Im}\left(\nu_{2}\right) \oplus \operatorname{Ker}\left(\mu_{3}\right) \cong \mathbb{Z}_{28} \oplus \mathbb{Z}_{4} \oplus \mathbb{Z}_{2}$. It follows from the presentation of $\pi_{0} \operatorname{Diff}\left(S^{3} \times S^{3}\right)$ given in Theorem 3 that this mapping class group is the factor group of the central extension of $\Gamma^{J}$ that corresponds to the cocycle $\omega_{1}-\omega_{2}$ of $H^{2}\left(\Gamma^{J}\right)$. It is clear that $\nu_{2}\left(\omega_{1}-\omega_{2}\right)$ will be a cocycle that corresponds to the group $\pi_{0} \operatorname{Diff}\left(S^{3} \times S^{3}\right)$. If we denote a generator of the summand $\mathbb{Z}_{2}$ by $\tilde{\omega}_{3}$, and generators of $\mathbb{Z}_{28}, \mathbb{Z}_{4}$ by $\tilde{\omega}_{1} \stackrel{\text { def }}{=} \nu_{2}\left(\omega_{1}\right)$ and $\tilde{\omega}_{2} \stackrel{\text { def }}{=} \nu_{2}\left(\omega_{2}\right)$ respectively, then these three cocycles $\tilde{\omega}_{i}, i \in\{1,2,3\}$ generate $H^{2}\left(\Gamma^{J}, \mathbb{Z}_{28}\right)$ and $\tilde{\omega}_{1}-\tilde{\omega}_{2}$ will be a cocycle that defines an extension isomorphic to $\pi_{0} \operatorname{Diff}\left(S^{3} \times S^{3}\right)$.

Remark. One can deduce from the proof of Theorem 5 that the cocycle $\tilde{\omega}_{3}$ defines the extension $E$ with $k_{0}=k_{1}=k_{2}=k_{3}=k_{4}=0$ and $k_{5}=k_{6}=1$.

We have shown that $\pi_{0} \operatorname{Diff}\left(S^{3} \times S^{3}\right)$ is an extension of $S L_{2}(\mathbb{Z})$ by $\mathcal{H}_{28}$. This extension defines a homomorphism $\zeta: S L_{2}(\mathbb{Z}) \longrightarrow O u t\left(\mathcal{H}_{28}\right)$. Using our Theorem 3 , we first find this homomorphism, i.e., we find $\zeta(y)$ and $\zeta(u)$ where $y$ and $u$ are the generators of $S L_{2}(\mathbb{Z})$ (see Lemma 1).

Recall that $\mathcal{H}_{28}$ is generated by three elements $A, B$ and $\Sigma$ and we can take elements $Y=(y, i d) ; \quad U=(u, i d) ; \Sigma=(i d, \Sigma) ; A=(i d, A) ; B=(i d, B) \epsilon$ $S L_{2}(\mathbb{Z}) \times \mathcal{H}_{28}$ to be generators of the mapping class group 1 The homomorphism $\zeta$ is defined by conjugation (in $\pi_{0} \operatorname{Diff}\left(S^{3} \times S^{3}\right)$ ), which means that

$$
g \circ \zeta(y)=\epsilon^{-1}\left(Y^{-1} \cdot \epsilon(g) \cdot Y\right) \quad \text { and } \quad g \circ \zeta(u)=\epsilon^{-1}\left(U^{-1} \cdot \epsilon(g) \cdot U\right)
$$

for any $g \in \mathcal{H}_{28}$ ( $\epsilon$ is the monomorphism of the exact sequence (1D).

Any element $g$ of $\mathcal{H}_{28}$ is a matrix

$$
g=B^{m} \cdot A^{n} \cdot \Sigma^{t}=\left(\begin{array}{ccc}
1 & n & t \\
0 & 1 & m \\
0 & 0 & 1
\end{array}\right) .
$$

The following identities can be easily verified using Theorem 3:

$$
\begin{gathered}
Y^{-1} A^{n} Y=(A B)^{n}, U^{-1} B^{m} U=\left(B A^{-1}\right)^{m},(A B)^{n}=A^{n} B^{n} \Sigma^{\frac{-n(n-1)}{2}}, \\
(B A)^{n}=B^{n} A^{n} \Sigma^{\frac{n(n-1)}{2}}, A^{n} B^{n}=B^{n} A^{n} \Sigma^{n^{2}},\left(B A^{-1}\right)^{m}=B^{m} A^{-m} \Sigma^{\frac{-m(m-1)}{2}} .
\end{gathered}
$$

These identities imply that

$$
\left(\begin{array}{ccc}
1 & n & t \\
0 & 1 & m \\
0 & 0 & 1
\end{array}\right) \circ \zeta(y)=\left(\begin{array}{ccc}
1 & n & t+\frac{n(n+1)}{2} \\
0 & 1 & m+n \\
0 & 0 & 1
\end{array}\right)
$$

and

$$
\left(\begin{array}{ccc}
1 & n & t \\
0 & 1 & m \\
0 & 0 & 1
\end{array}\right) \circ \zeta(u)=\left(\begin{array}{ccc}
1 & n-m & t-\frac{m(m-1)}{{ }^{2}} \\
0 & 1 & m^{2} \\
0 & 0 & 1
\end{array}\right) .
$$

These two formulas define the homomorphism $\zeta: S L_{2}(\mathbb{Z}) \longrightarrow \operatorname{Aut}\left(\mathcal{H}_{28}\right)$ (one can check that the map $\zeta$ respects the defining relations of the modular group).

\footnotetext{
${ }^{1}$ To avoid unnecessary notation, we use the same letters $A, B, \Sigma$ to denote the corresponding elements of the groups $\mathcal{H}_{28}$ and $\pi_{0} \operatorname{Diff}\left(S^{3} \times S^{3}\right)$.
} 
It is easy to see that the center of $\mathcal{H}_{28}$ is isomorphic to $\mathbb{Z}_{28}$ and it is a trivial $S L_{2}(\mathbb{Z})$-module. Hence there is a bijection between the set of equivalence classes of extensions giving rise to $\zeta$ and the elements of $H^{2}\left(S L_{2}(\mathbb{Z}), \mathbb{Z}_{28}\right)$ (see [5], Ch. IV, $\S 6)$. One can easily find that $H^{2}\left(S L_{2}(\mathbb{Z}), \mathbb{Z}_{28}\right) \cong \mathbb{Z}_{4}$.

To determine an element of this cohomology group corresponding to our mapping class group, we first describe a generator of $H^{2}\left(S L_{2}(\mathbb{Z}), \mathbb{Z}_{28}\right)$. Recall that the exact sequence of trivial $S L_{2}(\mathbb{Z})$-modules

$$
0 \longrightarrow \mathbb{Z} \stackrel{\times 28}{\longrightarrow} \mathbb{Z} \stackrel{\pi}{\longrightarrow} \mathbb{Z}_{28} \longrightarrow 0
$$

induces the following exact sequence in cohomology:

$$
H^{1}\left(S L_{2}(\mathbb{Z}), \mathbb{Z}_{28}\right) \longrightarrow H^{2}\left(S L_{2}(\mathbb{Z})\right) \stackrel{\times 28}{\longrightarrow} H^{2}\left(S L_{2}(\mathbb{Z})\right) \stackrel{\pi^{*}}{\longrightarrow} H^{2}\left(S L_{2}(\mathbb{Z}), \mathbb{Z}_{28}\right) .
$$

Hence we can take the cocycle $\varpi \stackrel{\text { def }}{=} \pi^{*}(f)$ to be a generator of $H^{2}\left(S L_{2}(\mathbb{Z}), \mathbb{Z}_{28}\right)$ where $f$ is the generator of $H^{2}\left(S L_{2}(\mathbb{Z})\right)$, defined above.

Lemma 8. The cocycle $-\varpi$ defines an extension $E_{-\varpi}: 0 \longrightarrow \mathcal{H}_{28} \longrightarrow E_{-\varpi} \longrightarrow$ $S L_{2}(\mathbb{Z}) \longrightarrow 0$ isomorphic to $\pi_{0} \operatorname{Diff}\left(S^{3} \times S^{3}\right)$.

Proof. As before, we write down a presentation of $E_{-\varpi}$ that coincides with the one of the mapping class group. We start with presentations of

$$
\mathcal{H}_{28} \cong\left\langle A, B, \Sigma \mid \Sigma^{28}=1, A \Sigma=\Sigma A, B \Sigma=\Sigma B, A B A^{-1} B^{-1}=\Sigma\right\rangle
$$

and

$$
\left.S L_{2}(\mathbb{Z}) \cong\langle y, u| y u y=u y u,(\text { yuy })^{4}=i d\right\rangle .
$$

Consider the lifting:

$$
Y=(y, i d) ; \quad U=(u, i d) \in S L_{2}(\mathbb{Z}) \times \mathcal{H}_{28} .
$$

We claim that the cocycle $-\varpi$ defines the relations that coincide with those of Theorem 3. First notice that the group law on the product $S L_{2}(\mathbb{Z}) \times \mathcal{H}_{28}$ is given as follows. For any $z, w \in S L_{2}(\mathbb{Z})$ and $g, f \in \mathcal{H}_{28}$,

$$
(z, g) \cdot(w, f)=(z w,-\varpi(z, w)[g \circ \zeta(w)] f) .
$$

Then one can easily find that $Y^{-1}=\left(y^{-1}, \Sigma\right)$ and $U^{-1}=\left(u^{-1}, \Sigma\right)$. Let us check that $B U=U B A^{-1}: U^{-1} B U=\left(u^{-1}, \Sigma\right) \cdot(i d, B) \cdot(u, i d)=\left(u^{-1}, \Sigma B\right) \cdot(u, i d)=$ $\left(i d, \Sigma^{-1}[\Sigma B \circ \zeta(u)]\right)$. Since

$$
\Sigma B \circ \zeta(u)=\left(\begin{array}{lll}
1 & 0 & 1 \\
0 & 1 & 1 \\
0 & 0 & 1
\end{array}\right) \circ \zeta(u)=\left(\begin{array}{ccc}
1 & -1 & 1 \\
0 & 1 & 1 \\
0 & 0 & 1
\end{array}\right)=\Sigma B A^{-1},
$$

we see that $U^{-1} B U=\left(i d, \Sigma^{-1}\left[\Sigma B A^{-1}\right]\right)=\left(i d, B A^{-1}\right)=(i d, B) \cdot\left(i d, A^{-1}\right)=$ $B A^{-1}$. Proofs of the other identities can be done in the same way and are left to the reader.

\section{ACKNOWLEDGEMENTS}

Results of this paper are part of the author's doctoral thesis written under the direction of Anatoly Libgober. I am deeply indebted to him for advice and continuing encouragement. 


\section{REFERENCES}

[1] R. Berndt and R. Schmidt: Elements of the Representation Theory of the Jacobi Group, Birkhäuser, Boston, 1998. MR 99i:11030

[2] J. Birman: On Siegel's modular group, Math. Ann. 191 (1971), 59-68. MR 43:6325

[3] W. Browder: Diffeomorphisms of 1-connected manifolds, Trans. Amer. Math. Soc. 128 (1967), 155-163. MR 35:3681

[4] W. Browder: Surgery on simply-connected manifolds, Springer-Verlag, New York, 1972. MR 50:11272

[5] K. Brown: Cohomology of Groups, Graduate Texts in Math., vol. 87, Springer-Verlag, New York, 1982. MR 83k:20002

[6] Y. Choie: A short note on the full Jacobi group, Proc. Amer. Math. Soc. 123 (1995), 26252627. MR 95k:11070

[7] J. Eells and N. Kuiper: An invariant for certain smooth manifolds, Annali di Math. 60 (1962), 93-110. MR 27:6280

[8] M. Eichler and D. Zagier: The Theory of Jacobi Forms, Birkhäuser, Boston, 1985. MR 86j:11043

[9] L. Evens: The Cohomology of Groups, Oxford Univ. Press, 1991. MR 93i:20059

[10] D. Fried: Word maps, isotopy and entropy, Trans. Amer. Math. Soc. 296 (1986), 851-859. MR 87k:58243

[11] G. Hochschild and J-P. Serre: Cohomology of group extensions, Trans. Amer. Math. Soc. 74 (1953), 110-134. MR 14:619b

[12] D. Johnson: A Survey of the Torelli Group, Contemporary Math. 20 (1983), 165-179. MR 85d:57009

[13] M. Kervaire and J. Milnor: Groups of homotopy spheres, Annals of Math. 77 (1963), 504-537. MR 26:5584

[14] M. Kreck: Isotopy classes of diffeomorphisms of $(k-1)$-connected almost parallelizable $2 k-$ manifolds, Algebraic Topology, Aarhus 1978, 643-663, Lecture Notes in Math., vol. 763, Springer, Berlin, 1979. MR 81i:57029

[15] B. Lawson and M. Michelson: Spin geometry, Princeton Math. Series, vol. 39, 1989. MR 91g:53001

[16] J. Milnor: Differentiable structures on spheres, American J. Math. 81 (1959), 962-972. MR 22:990

[17] J. Milnor: Lectures on the h-cobordism theorem, notes by L. Siebenmann and J. Sondow, Princeton University Press, 1965. MR 32:8352

[18] H. Sato: Diffeomorphism groups of $S^{p} \times S^{q}$ and exotic spheres, Quart. J. Math. Oxford (ser. 2) 20 (1969), 255-276. MR 40:6584

[19] E. Turner: A survey of diffeomorphism groups. Algebraic and geometrical methods in topology. Lecture Notes in Math. 428, 200-219, Springer-Verlag, Berlin-Heidelberg-New York, 1974. MR 52:4321

[20] B. Wajnryb: Mapping class group of a surface is generated by two elements, Topology 35, No. 2 (1996), 377-383. MR 96m:57007

Department of Mathematics, Statistics and Computer Science, University of Illinois at Chicago, 851 S. Morgan St., Chicago, Illinois 60607

E-mail address: krylov@math.uic.edu

Current address: School of Engineering and Science, International University Bremen, P. O.

Box 750 561, 28725 Bremen, Germany

E-mail address: n.krylov@iu-bremen.de 